\title{
The Markoff Group of Transformations in Prime and Composite Moduli
}

\author{
Chen Meiri and Doron Puder \\ with an Appendix by Dan Carmon
}

April 18, 2018

\begin{abstract}
The Markoff group of transformations is a group $\Gamma$ of affine integral morphisms, which is known to act transitively on the set of all positive integer solutions to the equation $x^{2}+y^{2}+z^{2}=x y z$. The fundamental strong approximation conjecture for the Markoff equation states that for every prime $p$, the group $\Gamma$ acts transitively on the set $X^{*}(p)$ of non-zero solutions to the same equation over $\mathbb{Z} / p \mathbb{Z}$. Recently, Bourgain, Gamburd and Sarnak proved this conjecture for all primes outside a small exceptional set.

In the current paper, we study a group of permutations obtained by the action of $\Gamma$ on $X^{*}(p)$, and show that for most primes, it is the full symmetric or alternating group. We use this result to deduce that $\Gamma$ acts transitively also on the set of non-zero solutions in a big class of composite moduli.

Our result is also related to a well-known theorem of Gilman and Evans, stating that for any finite non-abelian simple group $G$ and $r \geq 3$, the group Aut $\left(\mathrm{F}_{r}\right)$ acts on at least one " $T_{r}$-system" of $G$ as the alternating or symmetric group. In this language, our main result translates to that for most primes $p$, the group Aut $\left(\mathrm{F}_{2}\right)$ acts on a particular $T_{2}$-system of PSL $(2, p)$ as the alternating or symmetric group.
\end{abstract}

\section{Contents}

1 Introduction $\quad 2$

2 Preliminaries $\quad 6$

3 Alternating Group for $p \equiv 1(4) \quad 9$

4 Alternating Group for $p \equiv 3(4) \quad 9$

4.1 Primitivity of $Q_{p}$ when $p \equiv 3(4) \ldots \ldots \ldots \ldots \ldots$

4.2 Properties of blocks in the action of $Q_{p}$ on $Y^{*}(p) \ldots \ldots \ldots \ldots$

4.3 Deducing Alternating group from primitivity . . . . . . . . . . . . 17

5 Strong Approximation for Square Free Composite Moduli 19

5.1 Transitivity of $\Gamma$ on $Y^{*}(n) \ldots \ldots \ldots \ldots \ldots \ldots$

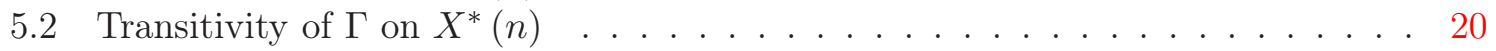

5.3 Transitivity without the classification . . . . . . . . . . . . . 24

$\begin{array}{lll}6 & T_{2} \text {-systems } & 27\end{array}$

A On the order of a quadratic integer modulo most primes

By Dan Carmon 


\section{Introduction}

The Markoff surface $\mathbb{X}$ is the affine surface in $\mathbb{A}^{3}$ defined by the equation ${ }^{1}$

$$
x^{2}+y^{2}+z^{2}=x y z .
$$

The Markoff triples $\mathcal{M}$ is the set of positive integer solutions to Equation (1), such as $(3,3,3)$. $\mathcal{M}$ The Markoff group of automorphisms of $\mathbb{X}$ is the group $\Gamma$ generated by permutations of the coordinates and the Vieta involutions $R_{1}, R_{2}$ and $R_{3}$ where $R_{3}(x, y, z)=(x, y, x y-z)$ and $R_{1}$ and $R_{2}$ are defined analogously. It is easy to see that $\mathcal{M}$ is invariant under $\Gamma$ and Markoff proved that $\Gamma$ acts transitively on $\mathcal{M}$ [Mar79, Mar80]. Let $\Delta$ be the group generated by $\Gamma$ and the involutions that replace two of the coordinates by their negatives. Then the set $\mathbb{X}(\mathbb{Z})$ of integer solutions to $(1)$ has two $\Delta$-orbits: $\{(0,0,0)\}$ and its complement $X^{*}(\mathbb{Z}) \stackrel{\text { def }}{=}$ $\mathbb{X}(\mathbb{Z}) \backslash\{(0,0,0)\}$.

\section{Prime Moduli}

If $p$ is a prime number, then $\mathbb{X}(\mathbb{Z} / p \mathbb{Z})$ is the finite set of solutions to (1) in $\mathbb{Z} / p \mathbb{Z}$, and we denote $X^{*}(p)=\mathbb{X}(\mathbb{Z} / p \mathbb{Z}) \backslash\{(0,0,0)\}$. The strong approximation conjecture for the Markoff equation (1) states that for every prime $p$, the reduction $\bmod p$ of the set of Markoff triples $\mathcal{M} \rightarrow X^{*}(p)$ is onto. This is clearly equivalent to $\Gamma$ acting transitively on $X^{*}(p)$. Recently, Bourgain, Gamburd and Sarnak proved this conjecture for all primes outside of a small exceptional set:

Theorem 1.1 (Bourgain-Gamburd-Sarnak [BGS17]). Let $E$ be the set of primes for which $\Gamma$ does not act transitively on $X^{*}(p)$. For any $\varepsilon>0$, the number of primes $p \leq T$ with $p \in E$ is at most $T^{\varepsilon}$, for $T$ large enough.

Moreover, for any $\varepsilon>0$, the largest $\Gamma$-orbit in $X^{*}(p)$ is of size at least $\left|X^{*}(p)\right|-p^{\varepsilon}$, for p large enough (whereas $\left|X^{*}(p)\right| \sim p^{2}$ ).

Let $\Gamma_{p}$ be the finite permutation group induced by the action of $\Gamma$ on $X^{*}(p)$. In the current work we study the nature of this group. The first step here is to notice that $\Gamma_{p}$ preserves a block structure as follows:

For $(x, y, z) \in X^{*}(p)$ denote by $[x, y, z]$ the block of all solutions obtained from $(x, y, z)$ by $[x, y, z]$ sign changes, so

$$
[x, y, z] \stackrel{\text { def }}{=}\{(x, y, z),(x,-y,-z),(-x, y,-z),(-x,-y, z)\} .
$$

Then $\Gamma_{p}$ preserves this block structure. Let $Y^{*}(p)$ denote the set of blocks in $X^{*}(p)$, and $Q_{p}$ denote the permutation group induced by the action of $\Gamma\left(\right.$ or $\left.\Gamma_{p}\right)$ on $Y^{*}(p)$. Simulations suggest the following conjecture:

Conjecture 1.2. For every $p \geq 5$, the permutation group $Q_{p}$ is the full alternating or symmetric group.

This conjecture was also raised, independently, in [CGMP16, Conjecture 1.3], where the authors also state precisely for which primes one can expect the alternating group $(p \equiv 3$ $\bmod 16)$ and for which the full symmetric group $(p \not \equiv 3 \bmod 16)$. If this conjecture holds, then roughly speaking (we give the precise formulation in Theorem 1.6 below), $\Gamma$ acts transitively on the solutions of (1) modulo $n$, for every square free.

Here we prove this conjecture for most primes. More particularly, we prove it for every $p \equiv 1(4)$ outside the exceptional set from Theorem 1.1, and for density-1 of the primes $p \equiv 3(4)$ :

\footnotetext{
${ }^{1}$ Sometimes the Markoff equation is written as $x^{2}+y^{2}+z^{2}=3 x y z$. However, these two equations are equivalent in the sense that their integer solutions are related bijectively by $(x, y, z) \longleftrightarrow(3 x, 3 y, 3 z)$. This bijection holds also for solutions in $\mathbb{Z} / p \mathbb{Z}$ for every prime $p \neq 3$.
} 
Theorem 1.3. If $p \equiv 1(4)$ and $Q_{p}$ is transitive, then $Q_{p}$ is the full alternating or symmetric group on $Y^{*}(p)$.

Namely, $Q_{p}$ is the full alternating or symmetric group for all $p \equiv 1(4)$ outside the exceptional set from Theorem 1.1. In fact, our proof yields that for every $p \equiv 1(4)$, the group $\Gamma$ acts as the full alternating or symmetric group on the large component described in Theorem 1.1. In the case $p \equiv 3(4)$, our proof is more involved and requires one further assumption:

Theorem 1.4. Let $p$ be a prime. Assume that:

- $p \equiv 3(4)$.

- $Q_{p}$ is transitive.

- The order of $\frac{3+\sqrt{5}}{2} \in \mathbb{F}_{p^{2}}$ is at least $32 \sqrt{p+1}$.

Then $Q_{p}$ is the full alternating or symmetric group on $Y^{*}(p)$.

The number $\frac{3+\sqrt{5}}{2}$ is related to the special solution $[3,3,3] \in Y^{*}(p)$ : its order inside $\mathbb{F}_{p^{2}}$ gives the length of the cycle of the transformation $[x, y, z] \mapsto[x, z, x z-y]$ containing the element $[3,3,3]$. For details see Sections 2 and 4.1.

As shown in Appendix A, the condition regarding the order of $\frac{3+\sqrt{5}}{2}$ is satisfied for density-1 of the primes ${ }^{2}$, hence

Corollary 1.5. For density-1 of all primes $p \equiv 3(4)$, the group $Q_{p}$ is the full alternating or symmetric group on $Y^{*}(p)$.

\section{Composite Moduli}

Let $n$ be a positive integer which is square-free, so $n=p_{1} \cdots p_{k}$ where $p_{1}, \ldots, p_{k}$ are distinct primes. Let $\mathbb{X}(n)$ denote the set of solutions to the Markoff equation (1) in $\mathbb{Z} / n \mathbb{Z}$. By the Chinese Remainder Theorem, $\mathbb{X}(n)=\mathbb{X}\left(p_{1}\right) \times \ldots \times \mathbb{X}\left(p_{k}\right)$, and let $X^{*}(n)=X^{*}\left(p_{1}\right) \times \ldots \times$ $X^{*}\left(p_{k}\right)$ be the set of solutions which are non-zero modulo any of the primes composing $n$. The action of $\Gamma$ on $\mathbb{X}(n)$ is the diagonal action on the $\mathbb{X}\left(p_{i}\right)$, and the subset $X^{*}(n)$ is invariant under this action. Denote the corresponding permutation group $\Gamma_{n}$. Is the action on $X^{*}(n) \Gamma_{n}$ transitive? It turns out that this would follow from Conjecture 1.2 and indeed holds true for the cases of that conjecture we establish:

Theorem 1.6. Let $n=p_{1} \cdots p_{k}$ be a product of distinct primes. If for every $j=1, \ldots, k$, $Q_{p_{j}} \geq \operatorname{Alt}\left(Y^{*}\left(p_{j}\right)\right)$, then $\Gamma$ acts transitively on $X^{*}(n)$.

In particular, if conjecture 1.2 holds, then $\Gamma$ acts transitively on $X^{*}(n)$ for every square-free $n$.

Corollary 1.7. Let $\mathcal{P}$ denote the set of primes that satisfy the assumptions of Theorem 1.3 or of Theorem 1.4. Then for every set of distinct primes $p_{1}, \ldots, p_{k} \in \mathcal{P}, \Gamma$ acts transitively on $X^{*}\left(p_{1} \cdots p_{k}\right)$.

Bourgain, Gamburd and Sarnak already proved Corollary 1.7 for primes $p \equiv 1$ (4) for which $\Gamma_{p}$ is transitive. This result should appear in the series announced in [BGS16]. We stress that our proof is entirely different: while Bourgain, Gamburd and Sarnak improve their techniques

\footnotetext{
${ }^{2}$ A set of primes $\mathcal{A}$ has density 1 if $\lim _{n \rightarrow \infty} \frac{\left|\mathcal{A} \cap \mathcal{P}_{n}\right|}{\left|\mathcal{P}_{n}\right|}=1$, where $\mathcal{P}_{n}=\{1<p \leq n \mid p$ is prime $\}$. In fact, the set of primes for which $\frac{3+\sqrt{5}}{2}$ has order at least $32 \sqrt{p+1}$ satisfies something slightly stronger than density $1-$ see Appendix A.
} 
from the proof of Theorem 1.1 so that the argument work for several primes simultaneously, our proof is group-theoretic and uses Theorem 1.1 as a black box. Both proofs rely on solutions containing the parabolic elements \pm 2 - see Figure 1 and Section 2.

For $n=p_{1} \cdots p_{k}$ as above, we use the notation $Y^{*}(n)=Y^{*}\left(p_{1}\right) \times \ldots \times Y^{*}\left(p_{k}\right)$ for the set of blocks in $X^{*}(n)$ and $Q_{n}$ for the permutation group induced by the action of $\Gamma$ on $Y^{*}(n)$. Note that these blocks are given by sign changes modulo every prime separately and are usually of size $4^{k}$ each (if all primes are odd). It is quite straight-forward to prove that under the assumptions of Theorem 1.6, $\Gamma$ acts transitively on $Y^{*}(n)$, using composition factors of $Q_{n}$. It requires some further argument to show that $\Gamma$ acts transitively on the full set $X^{*}(n)$. We elaborate in Section 5.

Remark 1.8 (Regarding the classification of finite simple groups). At this point we would like to remark on the dependence of our results on the Classification of Finite Simple Groups (CFSG). We use the classification only in the proof of Theorem 1.4: we first give an elementary proof that for a prime $p$ satisfying the assumptions in the theorem, $Q_{p}$ is a primitive permutation group $^{3}$, and then rely on (results depending on) the CFSG to deduce that $Q_{p}$ is the full alternating or symmetric group. If we rely on Theorem 1.6 to deduce Corollary 1.7, the latter also becomes partly dependent on the CFSG. This can be avoided, however, and to this aim we also give a proof that $\Gamma$ acts transitively on $X^{*}(n)$ assuming only that $Q_{p_{1}}, \ldots, Q_{p_{k}}$ are primitive permutation groups, without using the CFSG (see Theorem 1.9 below). To sum up, the only results depending on the CFSG are Theorem 1.4, Corollary 1.5, and the part of Theorem 1.11 relating to primes $p \equiv 3(4)$. In contrast, Theorems 1.3 and 1.6 and Corollary 1.7 do not depend on the CFSG. We illustrate this in Figure 1.

Indeed, the following result does not depend on the CFSG:

Theorem 1.9. Let $n=p_{1} \cdots p_{k}$ be a product of distinct primes. If $Q_{p_{1}}, \ldots, Q_{p_{k}}$ are primitive permutation groups, then $\Gamma$ acts transitively on $X^{*}(n)$.

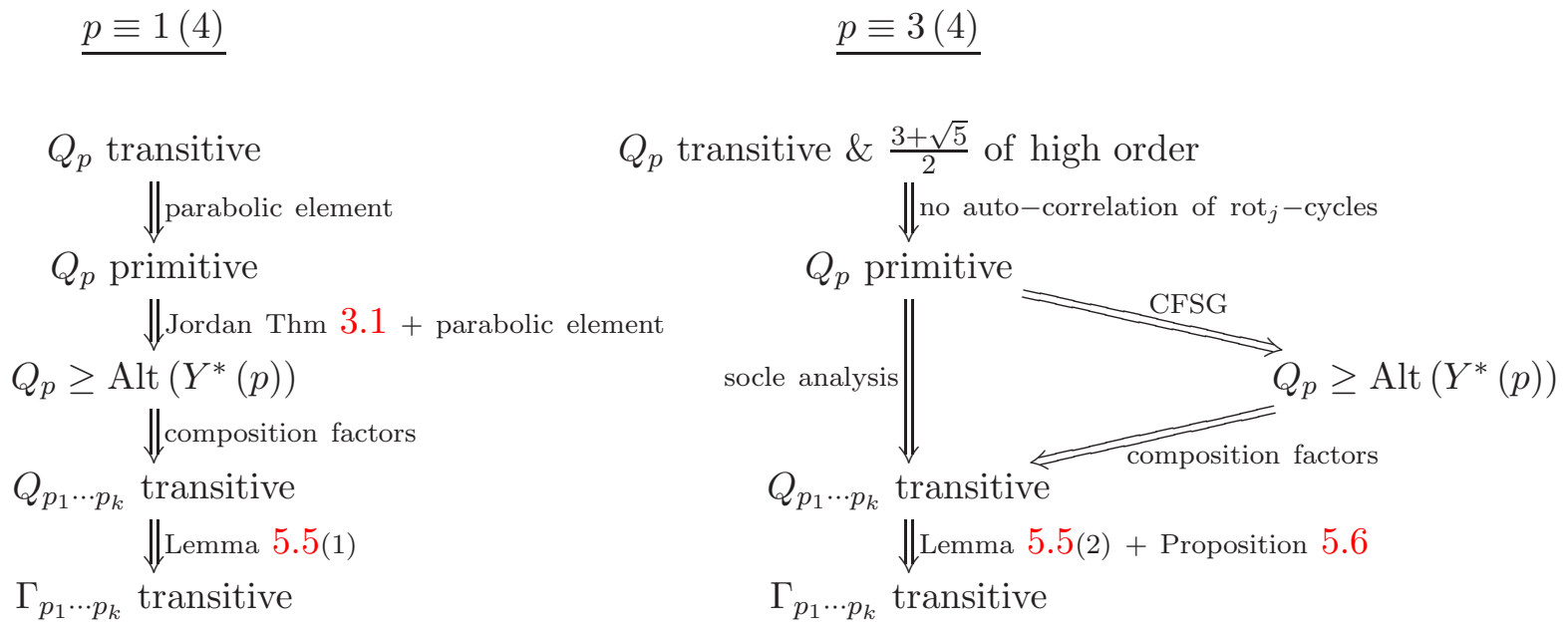

Figure 1: The flow of arguments in the paper. All the notions are explained in the sequel of the paper. Notice that the case $p \equiv 1(4)$ is indeed much simpler than its counterpart $p \equiv 3(4)$. To see the entire proof of the results for primes $p \equiv 1(4)$, it is enough to read Section 2, the short Section 3, the short Section 5.1 and Section 5.2 up to the the first half of Lemma 5.5.

\footnotetext{
${ }^{3}$ Recall that a permutation group $G \leq \operatorname{Sym}(m)$ is called primitive if it does not preserve any non-trivial block-structure. In particular, if $m \geq 3, G$ must be transitive.
} 


\section{$T_{2}$-systems}

Let $G$ be a finitely generated group and $\mathrm{F}_{r}$ the free group on $r$ generators. A normal subgroup $N \unlhd \mathrm{F}_{r}$ is said to be $G$-defining if $\mathrm{F}_{r} / N \cong G$. Denote by $\Sigma_{r}(G)$ the set of $G$-defining normal subgroups in $\mathrm{F}_{r}$. Consider the action of $\operatorname{Aut}\left(\mathrm{F}_{r}\right)$ (in fact, of Out $\left(\mathrm{F}_{r}\right)$ ) on $\Sigma_{r}(G)$. The orbits of this action are called $T_{r}$-systems of $G$.

The following theorem is due to Gilman (for $r \geq 4$ ) and Evans (who extended to $r=3$ ):

Theorem 1.10. [Gilyn, Eva93] Let $G$ be a finite non-abelian simple group and $r \geq 3$. Then $\operatorname{Aut}\left(\mathrm{F}_{r}\right)$ acts on at least one $T_{r}$-system of $G$ as the alternating or symmetric group.

In fact, Gilman and Evans provide more information about the special $T_{r}$-system on which Aut $\left(\mathrm{F}_{r}\right)$ acts as the full alternating or symmetric group, and show it is especially large. Gilman also showed that for $G=\operatorname{PSL}(2, \mathrm{p})$ with $p \geq 5$ prime, there is only one $T_{r}$-system for $r \geq 3$. Namely, he proved that Aut $\left(\mathrm{F}_{r}\right)$ acts transitively on $\Sigma_{r}(G)$. Theorem 1.10 says, of course, that the permutation group in this case is the alternating or symmetric group. For more details we refer the reader to the beautiful surveys [Pak01, Lub11].

When $r=2$, the action of Aut $\left(\mathrm{F}_{2}\right)$ on $\Sigma_{2}(G)$ is not transitive for any finite non-abelian simple group $G$. In fact, the number of $T_{2}$-systems tends to infinity as $|G| \rightarrow \infty$ [GS09]. The main reason for this phenomenon is that if $\{a, b\}$ are a set of generators of $\mathrm{F}_{2}$, and $\varphi: \mathrm{F}_{2} \rightarrow G$ an epimorphism, then the set of conjugacy classes of ${ }^{4} \varphi([a, b])$ and of $\varphi([a, b])^{-1}$ is a welldefined invariant of the $G$-defining subgroup $N=\operatorname{ker} \varphi$, which is also invariant under Aut $\left(\mathrm{F}_{2}\right)$. We elaborate more in Section 6.

Our result sheds more light on the case of $T_{2}$-systems for $G=\operatorname{PSL}(2, p)$. If $A, B \in \mathrm{SL}(2, p)$ and we denote $x=\operatorname{tr}(A), y=\operatorname{tr}(B)$ and $z=\operatorname{tr}(A B)$, then

$$
\operatorname{tr}([A, B])=x^{2}+y^{2}+z^{2}-x y z-2 .
$$

In Section 6 it is explained why the map $(A, B) \mapsto(\operatorname{tr}(A), \operatorname{tr}(B), \operatorname{tr}(A B))$ yields a bijection between the elements in $\Sigma_{2}(\operatorname{PSL}(2, \mathrm{p}))$ with associated trace -2 and the elements of $Y^{*}(p)$. In this language, the main result of [BGS17] - Theorem 1.1 above - says that outside the exceptional set of primes, these elements form a single $T_{2}$-system. See [MW13] for an extensive survey of the connection between the Markoff equation (1) and $T_{2}$-systems of PSL $(2, p)$. Through this connection, Theorems (1.3) and (1.4) translate to a result in the spirit of Theorem 1.10:

Theorem 1.11. Assume that the prime p satisfies the assumptions of Theorem 1.3 or of Theorem 1.4. Then Aut $\left(\mathrm{F}_{2}\right)$ acts on the trace- $(-2) T_{2}$-system of PSL $(2, p)$ as the full alternating or symmetric group.

The paper is organized as follows. Section 2 gives some more notation and collects some results from [BGS17] we use here. In the short Section 3 and longer Section 4 we prove Theorem 1.3 for $p \equiv 1(4)$ and Theorem 1.4 for $p \equiv 3(4)$, respectively. Section 5 is dedicated to proving the transitivity of $\Gamma$ in certain composite moduli: first assuming the groups $Q_{p}$ contain the alternating group (in Section 5.1), and then assuming only that $Q_{p}$ is primitive (Section 5.3). In Section 6 we give some background on $T$-systems and prove Theorem 1.11. Finally, Appendix A, by Dan Carmon, shows that the assumption in Theorem 4 regarding the order of $\frac{3+\sqrt{5}}{2} \in \mathbb{F}_{p^{2}}$ holds for most primes.

\footnotetext{
${ }^{4}$ Here, $[a, b]$ denotes the commutator $a b a^{-1} b^{-1}$.
} 


\section{Acknowledgments}

We are indebted to Peter Sarnak for his encouragement, and for stimulating discussions, enlightening suggestions and clever advice. We would also like to thank Zeev Rudnick and Pär Kurlberg for beneficial comments, and to Dan Carmon for writing the useful Appendix A. We have benefited much from the mathematical open source community, and in particular from SageMath. Author Meiri was supported by BSF grant 2014099 and ISF grant 662/15. Author Puder was supported by the Rothschild fellowship, by the NSF under agreement No. DMS1128155 and by the ISF grant 1071/16. Author Carmon was supported by the European Research Council under the European Union's Seventh Framework Programme (FP7/20072013) / ERC grant agreement $\mathrm{n}^{\mathrm{O}} 320755$.

\section{Preliminaries}

Before proving our main results, let us describe some further notation and collect further results from [BGS17] that we use below.

\section{Further notation}

- We already introduced above the notation $[x, y, z]$ for the block of the solution $(x, y, z)$ in $X^{*}(p)$, so $[x, y, z] \in Y^{*}(p)$. We also use this notation for a composite (square-free) modulo $n$ : here $[x, y, z]$ is the element (block) in $Y^{*}(n)$ containing the solution $(x, y, z)$.

- Some elements in $\Gamma$ are permutations of the three coordinates of solutions. We denote these elements by $\tau_{(12)}$ for the permutation exchanging the first and second coordinates, by $\tau_{(123)}$ for the cyclic permutation and so on. By abuse of notation, we use the same notation for the corresponding elements in $\Gamma, \Gamma_{p}, Q_{p}, \Gamma_{n}$ and $Q_{n}$.

- The analysis in [BGS17], as well as in the current work, relies heavily on three "rotation" elements $\operatorname{rot}_{1}, \operatorname{rot}_{2}, \operatorname{rot}_{3} \in \Gamma$. They are defined by

$$
\left.\operatorname{rot}_{j} \stackrel{\text { def }}{=} R_{j+2} \circ \tau_{(j+1} \quad j+2\right)
$$

(the indices are taken modulo 3 ). For example, $(x, y, z) \stackrel{\operatorname{rot}_{1}}{\mapsto}(x, z, x z-y)$. The rotation $\operatorname{rot}_{j}$ fixes the $j$-th coordinate and its action on $X^{*}(p)$ and on $Y^{*}(p)$ is completely analyzed in [BGS17] - see Lemmas 2.2 and 2.3 below. Again, by abuse of notation we write $\operatorname{rot}_{i}$ for the rotation element in the different groups $\Gamma, \Gamma_{p}, Q_{p}, \Gamma_{n}$ and $Q_{n}$.

- Following [BGS17], we denote the "conic sections" by $C_{j}(a), j=1,2,3$. These are $C_{j}(a)$ defined as

$$
C_{j}(a)=\left\{\left(x_{1}, x_{2}, x_{3}\right) \in X^{*}(p) \mid x_{j}=a\right\} .
$$

When we write $C_{j}( \pm a)$, we mean the conic section in $Y^{*}(p)$ :

$$
C_{j}( \pm a)=\left\{\left[x_{1}, x_{2}, x_{3}\right] \in Y^{*}(p) \mid x_{j}=a\right\} .
$$

- For every prime $p$ we let $i$ denote a square root of -1 (in $\mathbb{F}_{p}$ or in $\mathbb{F}_{p^{2}}$ ).

- For $x \in \mathbb{Z} / p \mathbb{Z}$ we use the standard Legendre symbol $\left(\frac{x}{p}\right)$ to denote the image of $x$ under ( $\left.\frac{x}{p}\right)$ the character of order 2. Namely,

$$
\left(\frac{x}{p}\right)= \begin{cases}1 & x \text { is a square in } \mathbb{F}_{\mathrm{p}}^{*} \\ -1 & x \text { is a non }- \text { square in } \mathbb{F}_{p}^{*} . \\ 0 & x=0\end{cases}
$$


- The notation $|x|$ is used to denote the order of the group element $x \in G$ in the group $G$.

\section{Rotation elements}

The action of $\operatorname{rot}_{1}$ on the conic section $C_{1}(x) \subseteq X^{*}(p)$ is a linear map on the last two coordinates given by the matrix

$$
\left(\begin{array}{cc}
0 & 1 \\
-1 & x
\end{array}\right)
$$

The eigenvalues of this matrix are given by $\frac{x \pm \sqrt{x^{2}-4}}{2}$. This leads to the following definitions and lemmas from [BGS17]:

Definition 2.1. $\quad$ - An element $x \in \mathbb{F}_{p}$ if called hyperbolic if $\left(x^{2}-4\right)$ is a square in $\mathbb{F}_{p}^{*}$.

hyperbolic

- An element $x \in \mathbb{F}_{p}$ if called elliptic if $\left(x^{2}-4\right)$ is a non-square in $\mathbb{F}_{p}^{*}$.

elliptic

- An element $x \in \mathbb{F}_{p}$ if called parabolic if $\left(x^{2}-4\right)=0$ in $\mathbb{F}_{p}$, namely, if $x= \pm 2$.

parabolic

Notice that this categorization of the elements is invariant under sign change $x \mapsto-x$. The following lemmas are based on Lemmas 3-5 of [BGS17] which describe the action of $\operatorname{rot}_{i}$ on $X^{*}(p)$. We adapt them below in order to describe the action of $\operatorname{rot}_{i}$ on $Y^{*}(p)$ and add some further details, all follow easily from Section 2.1 in [BGS17]. We state the lemmas for $C_{1}( \pm x)$, but the same statements holds, evidently, for $C_{2}( \pm x)$ and for $C_{3}( \pm x)$.

Lemma 2.2. [BGS17, Lemmas 3-5] Let $p \equiv 1(4)$ be prime. Then,

- $\left|Y^{*}(p)\right|=\frac{p(p+3)}{4}$.

- $\left|C_{1}( \pm 2)\right|=p$; The permutation induced by $\operatorname{rot}_{1}$ on $C_{1}( \pm 2)$ consists of a single $p$-cycle.

- There are $\frac{p-1}{4}$ hyperbolic elements up to sign. For $x$ hyperbolic, $\left|C_{1}( \pm x)\right|=\frac{p-1}{2}$. Let $\omega^{ \pm 1} \in \mathbb{F}_{p}$ be the eigenvalues of the matrix (2), so $x=\omega+\omega^{-1}$. The permutation induced by $\operatorname{rot}_{1}$ on $C_{1}( \pm x)$ consists of $\frac{p-1}{2 d}$ cycles of length $d$ each, where $d=\frac{\max (|\omega|,|-\omega|)}{2}$ and $|\omega|$ is the order of $\omega$ in the multiplicative group $\mathbb{F}_{p}^{*}$. The solutions in $C_{1}(x)$ have the form $\left(x, \alpha+\beta, \alpha \omega+\beta \omega^{-1}\right)$ for $\alpha, \beta \in \mathbb{F}_{p}^{*}$ with $\alpha \beta=\frac{x^{2}}{x^{2}-4}$, and

$$
\left(x, \alpha+\beta, \alpha \omega+\beta \omega^{-1}\right) \stackrel{\text { rot }_{1}}{\mapsto}\left(x, \alpha \omega+\beta \omega^{-1}, \alpha \omega^{2}+\beta \omega^{-2}\right) .
$$

- There are $\frac{p-1}{4}$ elliptic elements up to sign. For $x$ elliptic, $\left|C_{1}( \pm x)\right|=\frac{p+1}{2}$. Define $\omega$ as for hyperbolic elements by $x=\omega+\omega^{-1}$, only now $\omega \in \mathbb{F}_{p^{2}} \backslash \mathbb{F}_{p}$. The permutation induced by $\operatorname{rot}_{1}$ on $C_{1}( \pm x)$ consists of $\frac{p+1}{2 d}$ cycles of length $d$ each, where $d=\frac{\max (|\omega|,|-\omega|)}{2}$ and $|\omega|$ is the order of $\omega$ in the multiplicative group $\mathbb{F}_{p^{2}}^{*}$. Moreover, $\omega^{p+1}=1$, i.e. $|\omega| \mid(p+1)$. The solutions in $C_{1}(x)$ have the form $\left(x, A+A^{p}, A \omega+A^{p} \omega^{-1}\right)$ with $A \in \mathbb{F}_{p^{2}}^{*}$ and $A^{p+1}=\frac{x^{2}}{x^{2}-4}$, and

$$
\left(x, A+A^{p}, A \omega+A^{p} \omega^{-1}\right) \stackrel{\operatorname{rot}_{1}}{\mapsto}\left(x, A \omega+A^{p} \omega^{-1}, A \omega^{2}+A^{p} \omega^{-2}\right) .
$$

We sum up the content of Lemma 2.2 in Table 1.

When $p \equiv 3(4)$, our results are somewhat weaker and the proofs more involved. The main reason for that is the lack of solutions with the parabolic elements \pm 2 :

Lemma 2.3. [BGS17, Lemmas 3-5] Let $p \equiv 3$ (4) be prime. Then, 


\begin{tabular}{|c|c|c|c|c|}
\hline type of $x$ & $\begin{array}{l}\# x \text { 's up } \\
\text { to sign }\end{array}$ & $\left|C_{1}( \pm x)\right|$ & & cycle-structure for $\left.\operatorname{rot}_{1}\right|_{C_{1}( \pm x)}$ \\
\hline parabolic & 1 & $p$ & $x= \pm 2$ & a single $p$-cycle \\
\hline $\begin{array}{l}\text { hyperbolic } \\
\text { (including } \\
0 \text { ) }\end{array}$ & $\frac{p-1}{4}$ & $\frac{p-1}{2}$ & $\begin{array}{l}x=\omega+\omega^{-1} \\
\omega \in \mathbb{F}_{p}^{*} \backslash\{ \pm 1\}\end{array}$ & $\begin{array}{l}\text { For every } 1 \neq d \mid \frac{p-1}{2} \text {, there are }\left|\frac{\varphi(d)}{2}\right| \\
\text { hyperbolic } \pm x \text { such that }\left.\operatorname{rot}_{1}\right|_{C_{1}( \pm x)} \text { has } \\
\frac{p-1}{2 d} \text { cycles of length } d \text { each. (If } d \text { is odd, } \\
|w| \in\{d, 2 d\} \text {, if } d \text { is even, }|w|=2 d \text {.) }\end{array}$ \\
\hline elliptic & $\frac{p-1}{4}$ & $\frac{p+1}{2}$ & $\begin{array}{c}x=\omega+\omega^{-1} \\
\omega \in \\
\mathbb{F}_{p^{2} \backslash\{ \pm 1\}} \omega^{p+1}=1\end{array}$ & $\begin{array}{l}\text { For every } 1 \neq d \mid \frac{p+1}{2} \text {, there are } \frac{\varphi(d)}{2} \\
\text { elliptic } \pm x \text { such that }\left.\operatorname{rot}_{1}\right|_{C_{1}( \pm x)} \text { has } \frac{p+1}{2 d} \\
\text { cycles of length } d \text { each. }(|\omega| \in\{d, 2 d\})\end{array}$ \\
\hline
\end{tabular}

Table 1: The structure of $\operatorname{rot}_{1} \in Q_{p}$ when $p \equiv 1(4)$, as follows from Lemma 2.2. In the rightmost column, every set $\{x,-x\}$ is counted once.

\begin{tabular}{|c|c|c|c|c|}
\hline type of $x$ & $\begin{array}{l}\# x^{\prime} \text { s up } \\
\text { to sign }\end{array}$ & $\left|C_{1}( \pm x)\right|$ & $\begin{array}{c}\text { eigenvalues of } \\
\operatorname{rot}_{1}\end{array}$ & cycle-structure of $\left.\operatorname{rot}_{1}\right|_{C_{1}( \pm x)}$ \\
\hline $\begin{array}{l}\text { hyperbolic } \\
\left(\frac{x^{2}-4}{p}\right)=1\end{array}$ & $\frac{p-3}{4}$ & $\frac{p-1}{2}$ & $\begin{array}{c}\omega \in \mathbb{F}_{p}^{*} \backslash\{ \pm 1\} \\
x=\omega+\omega^{-1}\end{array}$ & $\begin{array}{c}\text { For every } 1 \neq d \mid \frac{p-1}{2} \text {, there are } \\
\frac{\varphi(d)}{2} \text { hyperbolic } \pm x \text { such that } \\
\left.\operatorname{rot}_{1}\right|_{C_{1}( \pm x)} \text { has } \frac{p-1}{2 d} \text { cycles of length } \\
d \text { each. }(|w| \in\{d, 2 d\})\end{array}$ \\
\hline $\begin{array}{c}\text { elliptic } \\
(\text { exc. } 0): \\
x \neq 0 \& \\
\left(\frac{x^{2}-4}{p}\right)=-1\end{array}$ & $\frac{p-3}{4}$ & $\frac{p+1}{2}$ & $\begin{array}{c}\omega \in \\
\mathbb{F}_{p^{2}}^{*} \backslash\{ \pm 1, \pm i\} \\
x=\omega+\omega^{-1} \\
\omega^{p+1}=1\end{array}$ & $\begin{array}{l}\text { For every } 3 \leq d \mid \frac{p+1}{2} \text {, there are } \\
\frac{\varphi(d)}{2} \text { elliptic } \pm x \text { such that } \\
\left.\operatorname{rot}_{1}\right|_{C_{1}( \pm x)} \text { has } \frac{p+1}{2 d} \text { cycles of length } \\
d \text { each. (If } d \text { is odd, }|\omega| \in\{d, 2 d\} \text {, if } \\
\quad d \text { is even, }|\omega|=2 d \text {.) }\end{array}$ \\
\hline
\end{tabular}

Table 2: The structure of $\operatorname{rot}_{1} \in Q_{p}$ when $p \equiv 3(4)$, as follows from Lemma 2.3

- $\left|Y^{*}(p)\right|=\frac{p(p-3)}{4}$

- There are no solutions in $Y^{*}(p)$ involving the parabolic elements \pm 2 , nor the elliptic element 0 .

- There are $\frac{p-3}{4}$ hyperbolic elements up to sign. For $x$ hyperbolic, the size and structure of $C_{1}( \pm x)$ and the action of $\operatorname{rot}_{1}$ on $C_{1}( \pm x)$ have the same properties as for $x$ hyperbolic when $p \equiv 1$ (4) (see Lemma 2.2).

- There are $\frac{p-3}{4}$ non-zero elliptic elements up to sign. For $x$ elliptic, the size and structure of $C_{1}( \pm x)$ and the action of $\operatorname{rot}_{1}$ on $C_{1}( \pm x)$ have the same properties as for $x$ elliptic when $p \equiv 1$ (4) (see Lemma 2.2).

We sum up the content of Lemma 2.3 in Table 2.

For $x \in \mathbb{F}_{p}$, denote by $d_{p}( \pm x)$ the order of $\operatorname{rot}_{1} \in Q_{p}$ in its action on $C_{1}( \pm x)$. Namely, $d_{p}( \pm x)$ the solutions with first coordinate $\pm x$ in $Y^{*}(p)$ belong to cycles of length $d_{p}( \pm x)$. 


\section{Alternating Group for $p \equiv 1(4)$}

This section contains the proof of Theorem 1.3, which states that if $p \equiv 1(4)$ and $Q_{p}$ is transitive, then $Q_{p}$ contains the entire alternating group Alt $\left(Y^{*}(p)\right)$. As mentioned above, the existence of parabolic elements when $p \equiv 1$ (4) allows a rather short argument in this case.

We use the following classical theorem of Jordan:

Theorem 3.1 (Jordan [DM96, Thm 3.3E]). Let $G \leq \operatorname{Sym}(n)$ be a primitive permutation group containing a cycle of prime length $p \leq n-3$. Then $G \geq \operatorname{Alt}(n)$.

Proof of Theorem 1.3. Assume $p \equiv 1(4)$, and let $\operatorname{rot}_{1} \in Q_{p}$ be the rotation element defined on Page 6. This element has one $p$-cycle, while all its other cycles have length coprime to $p$ (see Table 1). Thus its power $\sigma=\operatorname{rot}_{1}{ }^{\left|\operatorname{rot}_{1}\right| / p} \in Q_{p}$ is a $p$-cycle. As $\left|Y^{*}(p)\right|=\frac{p(p+3)}{4} \geq p+3$, it is now sufficient to show, by Jordan's Theorem (Theorem 3.1 above), that $Q_{p}$ is primitive in $\operatorname{Sym}\left(Y^{*}(p)\right)$.

We need to show that the group $Q_{p}$ preserves no non-trivial block structure. Assume there is a block structure $\left\{B_{1}, \ldots, B_{m}\right\}$ preserved by $Q_{p}$. So $\bigcup B_{i}=Y^{*}(p)$ and $B_{i} \cap B_{j}=\emptyset$ for $i \neq j$, and for every $g \in Q_{p}$ and every $i, g\left(B_{i}\right)=B_{j}$ for some $j$.

Consider $C_{1}( \pm 2) \subset Y^{*}(p)$, the $p$ elements contained in the cycle of size $p$ in $\sigma$. The set $C_{1}( \pm 2)$ must be contained in a block, for otherwise it has to be the union of several equallysized blocks, but $p$ is prime. Say $C_{1}( \pm 2) \subseteq B_{1}$. So $B_{1}$ contains all solutions with \pm 2 in the first coordinate. In particular, it contains $[2,2,2+2 i]$ and $[2,2+2 i, 2]$. But the same argument with $\operatorname{rot}_{2}$ and $\operatorname{rot}_{3}$ shows that $B_{1}$ contains all solutions with \pm 2 in any coordinate. So $B_{1}$ is invariant under all three rotations and under all permutations of coordinates, and therefore invariant under the action of the whole group $Q_{p}$. By the transitivity of $Q_{p}, B_{1}=Y^{*}(p)$.

Remark 3.2. The proof of Theorem 1.1 in [BGS17] shows that for every prime p, the large component of $X^{*}(p)$ contains all solutions with parabolic $( \pm 2)$ coordinates. Thus, our proof of Theorem 1.3 applies to the general case: the group $\Gamma$ acts on the large component of $Y^{*}(p)$ as the alternating or symmetric group.

\section{Alternating Group for $p \equiv 3(4)$}

In the case where $p \equiv 3(4)$, there are no parabolic elements, and in Sections 4.1 and 4.2 we establish the primitivity of $Q_{p}$ for density-1 of these primes rather than for all those outside the exceptional set from Theorem 1.1. We also rely on much deeper theorems, involving the classification of finite simple groups (CFSG), to conclude in Section 4.3 that whenever $Q_{p}$ is primitive, it contains Alt $\left(Y^{*}(p)\right)$. Throughout this section, we assume that $p \equiv 3(4)$.

\subsection{Primitivity of $Q_{p}$ when $p \equiv 3(4)$}

In this subsection we prove that under the assumptions of Theorem 1.4, the permutation group $Q_{p}$ is primitive. Namely,

Theorem 4.1. Let $p$ be prime with $p \equiv 3(4)$. Assume that $Q_{p}$ is transitive and that the order of $\frac{3+\sqrt{5}}{2} \in \mathbb{F}_{p^{2}}$ is at least $32 \sqrt{p+1}$. Then $Q_{p}$ is primitive.

To establish primitivity of $Q_{p}$, one needs to show there are no non-trivial blocks in the action of $Q_{p}$ on $Y^{*}(p)$ : a block is a subset $B \subseteq Y^{*}(p)$, such that for every $g \in Q_{p}$, either $g . B=B$ or $g \cdot B \cap B=\emptyset$. As $Q_{p}$ is assumed to be transitive, if $B$ is proper $\left(B \varsubsetneqq Y^{*}(p)\right)$ and

of size at least two, then the subsets $\left\{g . B \mid g \in Q_{p}\right\}$ constitute a partition of $Y^{*}(p)$ which is 
a non-trivial block structure preserved under the action of $Q_{p}$. So proving $Q_{p}$ is primitive is equivalent to showing that every proper block is a singleton.

The proof of Theorem 4.1 relies on the following two propositions which contain properties of blocks in $Y^{*}(p)$. We defer the proofs of these two propositions to the next subsection, and complete the proof of Theorem 4.1 in the current subsection, assuming the two propositions.

We say that some coordinate $j \in\{1,2,3\}$ is homogeneous in a block $B \subseteq Y^{*}(p)$ if the $j$-th coordinate of every solution in $B$ is of the same type (either all hyperbolic or all elliptic).

Proposition 4.2. Let $p \equiv 3(4)$. Assume that $Q_{p}$ acts transitively on $Y^{*}(p)$, and let $B \varsubsetneqq$ $Y^{*}(p)$ be a proper $Q_{p}$-block. Then at least two of the coordinates $\{1,2,3\}$ are homogeneous in $B$.

The most technical ingredient of the proof of primitivity is the following. Recall that $d_{p}( \pm x)$ denotes the length of the cycles of $\operatorname{rot}_{1} \in Q_{p}$ containing elements of $C_{1}( \pm x)$.

Proposition 4.3. Assume that $Q_{p}$ is transitive and let $x \in \mathbb{F}_{p} \backslash\{0, \pm 2\}$ satisfy $d_{p}( \pm x) \geq$ $16 \sqrt{p+1}$. Then, for every $j \in\{1,2,3\}$, every proper $Q_{p}$-block $B \varsubsetneqq Y^{*}(p)$ contains at most one solution with $j$-th coordinate $\pm x$.

The idea of the proof of this proposition is the following: assume there are two solutions in the block $B$ with first coordinate $\pm x$. Say these are $\left[x, y_{0}, y_{1}\right]$ and $\left[x, z_{0}, z_{1}\right]$. Then for every $1 \leq m$, the block $\operatorname{rot}_{1}{ }^{m}(B)$ contains the solutions $\left[x, y_{m}, y_{m+1}\right]$ and $\left[x, z_{m}, z_{m+1}\right]$ with $y_{m}$ and $z_{m}$ defined recursively by $y_{m+1}=x y_{m}-y_{m-1}$ and $z_{m+1}=x z_{m}-z_{m-1}$. By Proposition 4.2, at least one of the two coordinates 2,3 in every block is homogeneous, meaning that for every $m$, either $y_{m}$ and $z_{m}$ have the same type (hyperbolic or elliptic), or $y_{m+1}$ and $z_{m+1}$ have the same type. Using classical results in number theory, we show such "high correlation" between two cycles of $\operatorname{rot}_{1}$ is impossible whenever these cycles are long enough.

Section 4.2 gives the details of the proof, and assuming it, we finish the proof of Theorem 4.1. We need the following corollary showing that elements of high order in the sense of Proposition 4.3 appear in the same block and the same coordinate only with other elements of the same type and the same order:

Corollary 4.4. Assume that $Q_{p}$ is transitive and that $x \in \mathbb{F}_{p} \backslash\{0, \pm 2\}$ satisfies $d_{p}( \pm x) \geq$ $16 \sqrt{p+1}$. If $B \subsetneq Y^{*}(p)$ is a proper $Q_{p}$-block containing some solution with first coordinate $\pm x$, and another solution with first coordinate $\pm x^{\prime}$, then $d_{p}( \pm x)=d_{p}\left( \pm x^{\prime}\right)$. In particular, $x$ and $x^{\prime}$ are of the same type (both hyperbolic or both elliptic).

Proof. Note that $\operatorname{rot}_{1}{ }^{d_{p}( \pm x)}(B)=B$. By Proposition $4.3, \operatorname{rot}_{1}^{m}(B) \neq B$ for $1 \leq m<d_{p}( \pm x)$. Hence, $d_{p}\left( \pm x^{\prime}\right)$ is some multiple of $d_{p}( \pm x)$. In particular, the assumption of Proposition 4.3 holds for $x^{\prime}$, and by symmetry, $d_{p}( \pm x)$ is a multiple of $d_{p}\left( \pm x^{\prime}\right)$. Hence $d_{p}\left( \pm x^{\prime}\right)=d_{p}( \pm x)$.

Proof of Theorem 4.1 assuming Propositions 4.2 and 4.3. Assume that $Q_{p}$ is transitive and $\omega=\frac{3+\sqrt{5}}{2} \in \mathbb{F}_{p^{2}}^{*}$ has order at least $32 \sqrt{p+1}$. We need to show that $Q_{p}$ is primitive. We use the special symmetric solution $[3,3,3] \in Y^{*}(p)$. Whenever $\omega \in \mathbb{F}_{p^{2}}$ has high order in the multiplicative group $\mathbb{F}_{p^{2}}^{*}$, the cycle of $\operatorname{rot}_{1} \in Q_{p}$ containing the solution $[3,3,3]$ is long. More concretely, $3=\omega+\omega^{-1}$, and by Lemma 2.3 and Table $2, d_{p}( \pm 3)$ is either $|\omega|$ or $\frac{|\omega|}{2}$, where $|\omega|$ is the order of $\omega$ in the multiplicative group $\mathbb{F}_{p^{2}}{ }^{*}$. So $d_{p}( \pm 3) \geq 16 \sqrt{p+1}$.

Assume that $[a, b, c]$ and $[3,3,3]$ are two distinct solutions lying in the same proper $Q_{p^{-}}$ block $B \varsubsetneqq Y^{*}(p)$. By Lemma 2.3, $d_{p}( \pm 3) \geq 16 \sqrt{p+1}$, and by Corollary $4.4, d_{p}( \pm a)=$ $d_{p}( \pm b)=d_{p}( \pm c)=d_{p}( \pm 3)$. As $[3,3,3]$ is the only solution of the form $[x, x, x]$ or $[x, x,-x]$, we can assume without loss of generality that $\{ \pm b\} \neq\{ \pm c\}$. Since $\left.\tau_{(2} 3\right)$ stabilizes $[3,3,3]$, we have $\tau_{(23)}(B)=B$, so the two distinct solutions $[a, b, c]$ and $[a, c, b]$ both belong to $B$. This 
contradicts Proposition 4.3: $d_{p}( \pm a)=d_{p}( \pm 3)$ is large and thus $a$ cannot appear twice in the same coordinate in the same block.

As mentioned in Section 1, the assumptions in Theorem 4.1 hold for density-1 of the primes $p \equiv 3$ (4). Indeed, relying on strong results of Ford [For08], Dan Carmon proves in Proposition A.1 in Appendix A that under some assumptions, the order of a quadratic integer modulo primes is high for density-1 of the primes. From Proposition A.1 we deduce:

Corollary 4.5. For density- 1 of all primes, the element $\omega=\frac{3+\sqrt{5}}{2} \in \mathbb{F}_{p^{2}}$ has order at least $32 \sqrt{p+1}$ in the multiplicative group $\mathbb{F}_{p^{2}}{ }^{*}$, in which case $d_{p}( \pm 3) \geq 16 \sqrt{p+1}$.

Combining Theorem 1.1 with Corollary 4.5 shows why the assumptions in Theorem 4.1 hold for density-1 of all primes $p \equiv 3(4)$, hence:

Corollary 4.6. For density-1 of all primes $p \equiv 3(4)$, the group $Q_{p}$ is primitive in its action on $Y^{*}(p)$.

Remark 4.7. It is conceivable that there is a stronger version of Proposition 4.3 which states there cannot be correlation between two long cycles of $\operatorname{rot}_{1} \in Q_{p}$ even with two different first coordinates. Were we able to prove this, we could omit the condition about the order of $\frac{3+\sqrt{5}}{2}$ in the statements of Theorems 1.4 and 4.1 and assume only that $Q_{p}$ is transitive to conclude that it is primitive and, moreover, contains Alt $\left(Y^{*}(p)\right.$ ). (This would make Theorem 1.4 completely parallel to Theorem 1.3 dealing with $p \equiv 1(4)$.) In Remark 4.12 below we explain the obstacle to proving this more general version of Proposition 4.3.

\subsection{Properties of blocks in the action of $Q_{p}$ on $Y^{*}(p)$}

In the current subsection we prove the two propositions that were stated without proof in the previous subsection. Proposition 4.2 is proved in Section 4.2.1, and Proposition 4.3 proved in Sections 4.2.2 (the hyperbolic case) and 4.2.3 (the elliptic case).

\subsubsection{Homogeneity of coordinates in blocks}

Lemma 4.8. The subgroup $H=\left\langle\operatorname{rot}_{1}, \operatorname{rot}_{2}, \operatorname{rot}_{3}\right\rangle \leq \Gamma$ has index at most 2 in $\Gamma$.

Proof. By definition, $\Gamma$ is generated by the three Vieta involutions and permutations of coor-

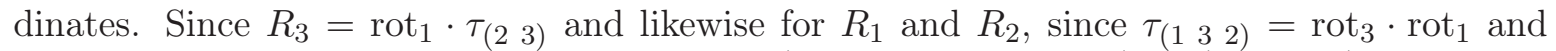
since $S_{3}=\langle(12),(132)\rangle$, we obtain that $\Gamma=\left\langle\operatorname{rot}_{1}, \operatorname{rot}_{2}, \operatorname{rot}_{3}, \tau_{(12)}\right\rangle=\left\langle H, \tau_{(12)}\right\rangle$. It is easy

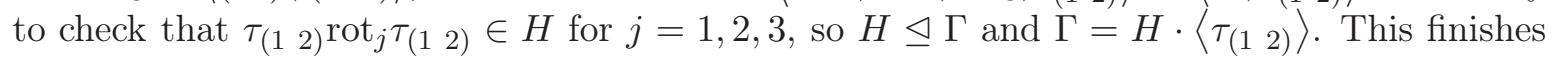
the proof.

Recall that Proposition 4.2 says that if $Q_{p}$ acts transitively on $Y^{*}(p)$, and if $B \varsubsetneqq Y^{*}(p)$ is a proper block of the action of $Q_{p}$ on $Y^{*}(p)$, then at least two of the coordinates $\{1,2,3\}$ are homogeneous in $B$.

Proof of Proposition 4.2. Assume that some coordinate, say $j=1$, is not homogeneous in $B$. We need to show that the second and third coordinates are homogeneous. The element $\operatorname{rot}_{1}^{(p-1) / 2}$ fixes every solution with first coordinate hyperbolic, while $\operatorname{rot}_{1}^{(p+1) / 2}$ fixes every solution with first coordinate elliptic. Hence $B$ is invariant under both elements, and thus by rot $_{1}$.

By the same argument, if all three coordinates are not homogeneous, $B$ is invariant under $H_{p}=\left\langle\operatorname{rot}_{1}, \operatorname{rot}_{2}, \operatorname{rot}_{3}\right\rangle \leq Q_{p}$. By Lemma 4.8, $\left[Q_{p}: H_{p}\right] \leq 2$, and transitivity implies there are at most two blocks in the action: $B$ and $B^{\prime}=\gamma(B)$ for some $\gamma \in Q_{p}$. But the block 
containing $[3,3,3]$ is also invariant under $\tau_{(12)}$, hence is invariant under the whole of $Q_{p}-\mathrm{a}$ contradiction.

Thus at least one coordinate - the second or the third - is homogeneous. Notice that $\operatorname{rot}_{1}$, which stabilizes $B$, moves the third coordinate of the solutions to the second. Hence both the second and third coordinates must be homogeneous.

Remark 4.9. In fact, the proof of the last lemma yields something slightly stronger. Denote the type of a solution in $Y^{*}(p)$ by some triple in $\{h, e\}^{3}$, depending on whether every coordinate is hyperbolic or elliptic. Then, every block $B$ as above contains either only solutions of the same type (homogeneous in all coordinates), or only solutions of exactly two types: one type is $(h, h, h)$ or $(e, e, e)$, and the other differs from the first type in one coordinate (the sole non-homogeneous coordinate).

\subsubsection{No correlation between two long $\operatorname{rot}_{1}$-cycles with the same first hyperbolic coordinate}

We now prove Proposition 4.3 stating that if $Q_{p}$ is transitive and $d_{p}( \pm x) \geq 16 \sqrt{p+1}$, then $\pm x$ cannot appear twice in the same coordinate in the same proper $Q_{p}$-block $B \varsubsetneqq Y^{*}(p)$. What we actually prove is the lack of correlation between two long enough cycles of $\operatorname{rot}_{j}$ with the same $j$-th coordinate (including the case of two different offsets of the same cycle). The proof of Proposition 4.3 is split to the case where $x$ is hyperbolic (in the current subsection) and the case it is elliptic (given in Section 4.2.3).

We use the following classical number-theoretic result:

Theorem 4.10 (Weil [Sch76, Theorem II.2C']). Let $f(x) \in \mathbb{F}_{p}[x]$ be a polynomial with $m$ distinct roots in $\overline{\mathbb{F}_{p}}$ which is not a square in $\overline{\mathbb{F}_{p}}[x]$. Then

$$
\left|\sum_{s \in \mathbb{F}_{p}}\left(\frac{f(s)}{p}\right)\right| \leq(m-1) \sqrt{p} .
$$

Proof of Proposition 4.3 when $x$ is hyperbolic. Assume that $x$ is hyperbolic with $d_{p}( \pm x) \geq$ $16 \sqrt{p+1}$, and that there are two elements in the proper $Q_{p}$-block $B \varsubsetneqq Y^{*}(p)$ with $\pm x$ in the first coordinate. The same arguments holds, evidently, for every coordinate $j=1,2,3$.

Assume that $\left[x, y_{0}, y_{1}\right]$ and $\left[x, z_{0}, z_{1}\right]$ belong to $B$. By Lemma $2.3, x=\omega+\omega^{-1}$ with $\omega \in \mathbb{F}_{p}^{*}$ and we can assume $|\omega|=2 d \geq 32 \sqrt{p-1}$ : otherwise, replace $x$ with $-x$ and $\omega$ with $-\omega$. Write $y_{0}=\alpha+\beta$ with $\alpha, \beta \in \mathbb{F}_{p}^{*}$ so that $\alpha \beta=\frac{x^{2}}{x^{2}-4}$ and $y_{1}=\alpha \omega+\beta \omega^{-1}$ (see Lemma 2.3). The cycle of rot $_{1}$ containing $\left[x, y_{0}, y_{1}\right]$ is

$$
\left[x, y_{0}, y_{1}\right]=\left[x, y_{d}, y_{d+1}\right],\left[x, y_{1}, y_{2}\right], \ldots,\left[x, y_{d-2}, y_{d-1}\right],\left[x, y_{d-1}, y_{d}\right]
$$

with

$$
y_{j}=\alpha \omega^{j}+\beta \omega^{-j} .
$$

The set $\left\{\omega^{j}\right\}_{0 \leq j \leq 2 d-1}$ is the same as the set $\left\{s^{m}\right\}_{s \in \mathbb{F}_{p}^{*}}$ where $m=\frac{p-1}{2 d}$ (with every element in $\left\{\omega^{j}\right\}$ covered by $\frac{p-1}{2 d}$ different values of $s$ ). So as sets,

$$
\left\{y_{0}, \ldots, y_{2 d-1}\right\}=\left\{\alpha \omega^{j}+\beta \omega^{-j}\right\}_{0 \leq j \leq 2 d-1}=\left\{f_{\alpha, \beta}(s) \stackrel{\text { def }}{=} \alpha s^{m}+\beta s^{-m}\right\}_{s \in \mathbb{F}_{p}^{*}} .
$$

The same holds for the cycle of $\operatorname{rot}_{1}$ containing $\left[x, z_{0}, z_{1}\right]$ with $\gamma, \delta \in \mathbb{F}_{p}^{*}$ in the role of $\alpha, \beta$, so that $z_{j}=\gamma \omega^{j}+\delta \omega^{-j}$. We may assume that $\gamma \neq \pm \alpha$, for otherwise $\left[x, y_{0}, y_{1}\right]=\left[x, z_{0}, z_{1}\right]$. Moreover, if $s^{m}=\omega^{j}$ then $f_{\alpha, \beta}(s)=y_{j}$ and $f_{\gamma, \delta}(s)=z_{j}$. 
Notice that $y_{j}$ and $z_{j}$ are of different types (one hyperbolic and the other elliptic) if and only if

$$
\left(\frac{\left(y_{j}^{2}-4\right)\left(z_{j}^{2}-4\right)}{p}\right)=-1
$$

Since $\left[x, y_{j}, y_{j+1}\right]$ and $\left[x, z_{j}, z_{j+1}\right]$ both belong to the block $\operatorname{rot}_{1}^{j}(B)$, we derive from Proposition 4.2 that (5) cannot hold for two consecutive values of $j$. In the parametrization given by $s \in \mathbb{F}_{p}^{*}$, this means that

$$
\left(\frac{\left(f_{\alpha, \beta}(s)^{2}-4\right)\left(f_{\gamma, \delta}(s)^{2}-4\right)}{p}\right)=\left(\frac{\left(f_{\alpha \omega, \beta \omega^{-1}}(s)^{2}-4\right)\left(f_{\gamma \omega, \delta \omega^{-1}}(s)^{2}-4\right)}{p}\right)=-1
$$

cannot hold for any $s \in \mathbb{F}_{p}^{*}$.

Write

$$
g_{\alpha, \beta}(s) \stackrel{\text { def }}{=}\left(s^{m}\right)^{2}\left(f_{\alpha, \beta}(s)^{2}-4\right)=\left[\left(\alpha s^{2 m}+\beta\right)^{2}-4 s^{2 m}\right] \in \mathbb{F}_{p}[s],
$$

and $k_{1}(s)=g_{\alpha, \beta}(s) g_{\gamma, \delta}(s)$ and $k_{2}(s)=g_{\alpha \omega, \beta \omega^{-1}}(s) g_{\gamma \omega, \delta \omega^{-1}}(s)$. Now (6) is equivalent to

$$
\left(\frac{k_{1}(s)}{p}\right)=\left(\frac{k_{2}(s)}{p}\right)=-1
$$

Denote by $N_{(-1,-1)}$ the number of $s \in \mathbb{F}_{p}$ for which $(7)$ holds. Our goal is to show that $N_{(-1,-1)}>0$, whence $(7)$ has some solution $s \neq 0$, yielding a contradiction (note that $s=0$ is not a solution to $(7))$. Note that $k_{1}(s), k_{2}(s) \neq 0$ for every $s \in \mathbb{F}_{p}$ : indeed, $g_{\alpha, \beta}(0)=\beta^{2} \neq 0$, and if $0 \neq s \in \mathbb{F}_{p}$ and $g_{\alpha, \beta}(s)=0$ then $f_{\alpha, \beta}(s)= \pm 2$ is $y_{j}$ for some $j$, but there are no solution in $X^{*}(p)$ containing \pm 2 when $p \equiv 3(4)$. Therefore $\left(\frac{k_{1}(s)}{p}\right),\left(\frac{k_{2}(s)}{p}\right) \neq 0$ for $s \in \mathbb{F}_{p}$ and

$$
\begin{aligned}
N_{(-1,-1)} & =\frac{1}{4} \sum_{s \in \mathbb{F}_{p}}\left(1-\left(\frac{k_{1}(s)}{p}\right)\right)\left(1-\left(\frac{k_{2}(s)}{p}\right)\right) \\
& =\frac{1}{4}\left[p-\sum_{p \in \mathbb{F}_{p}}\left(\frac{k_{1}(s)}{p}\right)-\sum_{p \in \mathbb{F}_{p}}\left(\frac{k_{2}(s)}{p}\right)+\sum_{p \in \mathbb{F}_{p}}\left(\frac{k_{1}(s) k_{2}(s)}{p}\right)\right] .
\end{aligned}
$$

For every $\emptyset \neq B \subseteq\{1,2\}$, define

$$
M_{B} \stackrel{\text { def }}{=} \sum_{s \in \mathbb{F}_{p}}\left(\frac{\prod_{j \in B} k_{j}(s)}{p}\right)
$$

Then (8) becomes

$$
N_{(-1,-1)}=\frac{1}{4}\left[p-M_{\{1\}}-M_{\{2\}}+M_{\{1,2\}}\right] .
$$

We use Theorem 4.10 to estimate the $M_{B}$ 's. First, we show that none of $k_{1}, k_{2}$ and $k_{1} k_{2}$ are squares in $\overline{\mathbb{F}_{p}}[x]$. The roots of

$$
g_{\alpha, \beta}(s)=\left(\alpha s^{2 m}+\beta+2 s^{m}\right)\left(\alpha s^{2 m}+\beta-2 s^{m}\right)
$$

satisfy

$$
s^{m}=\frac{ \pm 2 \pm \sqrt{4-4 \alpha \beta}}{2 \alpha}=\frac{ \pm 1 \pm \sqrt{1-\frac{x^{2}}{x^{2}-4}}}{\alpha}=\frac{ \pm 1 \pm \sqrt{\frac{-4}{x^{2}-4}}}{\alpha} .
$$


As $x$ is hyperbolic and $p \equiv 3(4)$, we have that $\frac{-4}{x^{2}-4}$ is not a square in $\mathbb{F}_{p}$, so 1 and $\sqrt{\frac{-4}{x^{2}-4}}$ are linearly independent over $\mathbb{F}_{p}$, and $\frac{ \pm 1 \pm \sqrt{\frac{-4}{x^{2}-4}}}{\alpha}$ are four distinct values for $S^{m}$, different from zero. Moreover, the polynomial $s^{m}-\xi$ is separable for $0 \neq \xi \in \mathbb{F}_{p^{2}}$ because $m=\frac{p-1}{2 d}<p$. So $g_{\alpha, \beta}(s)$, which is of degree $4 m$, has $4 m$ distinct roots in $\overline{\mathbb{F}_{p}}$, and in particular is not a square in $\overline{\mathbb{F}_{p}}[x]$.

This analysis shows that $g_{\alpha, \beta}$ and $g_{\gamma, \delta}$ have a common root if and only if $\alpha= \pm \gamma$. Since $\alpha \neq \pm \gamma$ by assumption, $k_{1}=g_{\alpha, \beta} g_{\gamma, \delta}$ and $k_{2}=g_{\alpha \omega, \beta \omega^{-1}} g_{\gamma \omega, \delta \omega^{-1}}$ are both separable of degree $8 m$. Finally, $k_{1} k_{2}$, of degree $16 m$, is also not a square in $\overline{\mathbb{F}_{p}}[x]$ : for $\alpha \neq \pm \alpha \omega$ and if $\alpha= \pm \gamma \omega$ then $\alpha \omega \neq \pm \gamma$.

Theorem 4.10 yields that $\left|M_{\{1\}}\right|,\left|M_{\{2\}}\right| \leq(8 m-1) \sqrt{p}$ and $\left|M_{\{1,2\}}\right| \leq(16 m-1) \sqrt{p}$. From (10) we now obtain

$$
\begin{array}{rll}
N_{(-1,-1)} & \geq & \frac{1}{4}[p-2(8 m-1) \sqrt{p}-(16 m-1) \sqrt{p}] \\
& = & \frac{1}{4}[p-32 m \sqrt{p}+3 \sqrt{p}] \\
& =\frac{p-1}{2 d} & \frac{1}{4}\left[p-\frac{16(p-1)}{d} \sqrt{p}+3 \sqrt{p}\right] \\
d \geq 16 \sqrt{p-1} & \frac{1}{\geq}[p-\sqrt{(p-1) p}+3 \sqrt{p}]>\frac{3 \sqrt{p}}{4}>0 .
\end{array}
$$

\subsubsection{No correlation between two long $\operatorname{rot}_{1}$-cycles with the same first elliptic coordinate}

The general proof strategy for the elliptic case is the same as for the hyperbolic case, albeit with a few extra technical details. In the hyperbolic case, we used a parametrization of the elements of a cycle of $\operatorname{rot}_{1}$ as a function over $\mathbb{F}_{p}^{*}$, which allowed us to use Weil's bound (Theorem 4.10 above). In the elliptic case, a similar approach requires that we go over the elements in the cyclic subgroup of size $p+1$ in $\mathbb{F}_{p^{2}}^{*}$. The following lemma allows us to parametrize this subgroup as a function over $\mathbb{F}_{p}$ :

Lemma 4.11. The multiplicative subgroup $H \leq \mathbb{F}_{p^{2}}^{*}$ of order $p+1$ satisfies

$$
H=\left\{\theta+i \eta \mid \theta, \eta \in \mathbb{F}_{p}, \theta^{2}+\eta^{2}=1\right\}=\left\{\frac{2 s}{1+s^{2}}+i \frac{1-s^{2}}{1+s^{2}} \mid s \in \mathbb{F}_{p}\right\} \cup\{-i\}
$$

(where $i=\sqrt{-1} \in \mathbb{F}_{p^{2}}$ ).

Proof. Note that $(\theta+i \eta)^{p}=\theta-i \eta$ (recall that $p \equiv 3(4)$ so $i^{p}=i^{4 k+3}=i^{3}=-i$ ). So $(\theta+i \eta)^{p+1}=(\theta+i \eta)(\theta-i \eta)=\theta^{2}+\eta^{2}$. This gives the first equality in (11). A straightforward computation yields the second equality.

Proof of Proposition 4.3 when $x$ is elliptic. We assume that $x$ is elliptic with $d_{p}( \pm x) \geq 16 \sqrt{p+1}$, and assume that there are two elements in the proper $Q_{p}$-block $B \varsubsetneqq$ $Y^{*}(p)$ with $\pm x$ in the first coordinate. We use the notation $H$ for the subgroup of order $p+1$ in $\mathbb{F}_{p^{2}}^{*}$, as in Lemma 4.11. Assume that $\left[x, y_{0}, y_{1}\right]$ and $\left[x, z_{0}, z_{1}\right]$ both belong to $B$. By Table 2 , $x=\omega+\omega^{-1}$ with $\omega \in H$, and we can assume that $|\omega|=2 d \geq 32 \sqrt{p+1}$, for otherwise replace 
$\omega$ by $-\omega$ and $x$ by $-x$. Let $A \in \mathbb{F}_{p^{2}}$ satisfy that $A^{p+1}=\frac{x^{2}}{x^{2}-4}$, that $y_{0}=A+A^{p}$ and that $y_{1}=A \omega+A^{p} \omega^{-1}$ (see Lemma 2.3). The cycle of $\operatorname{rot}_{1}$ containing $\left[x, y_{0}, y_{1}\right]$ is

$$
\left[x, y_{0}, y_{1}\right]=\left[x, y_{d}, y_{d+1}\right],\left[x, y_{1}, y_{2}\right], \ldots,\left[x, y_{d-2}, y_{d-1}\right],\left[x, y_{d-1}, y_{d}\right]
$$

with

$$
y_{j}=A \omega^{j}+A^{p} \omega^{-j}
$$

The set $\left\{\omega^{j}\right\}_{0 \leq j \leq 2 d-1}$ is the same as the set $\left\{h^{m}\right\}_{h \in H}$ where $m=\frac{p+1}{2 d}$, with every element in $\left\{\omega^{j}\right\}$ covered by $m$ different values of $h$. So as sets,

$$
\left\{y_{0}, \ldots, y_{2 d-1}\right\}=\left\{A \omega^{j}+A^{p} \omega^{-j}\right\}_{0 \leq j \leq 2 d-1}=\left\{f_{A}(h) \stackrel{\text { def }}{=} A h^{m}+A^{p} h^{-m}\right\}_{h \in H} .
$$

The same holds for the cycle of $\operatorname{rot}_{1}$ containing $\left[x, z_{0}, z_{1}\right]$ with $C \in \mathbb{F}_{p^{2}}$ in the role of $A$, so that $z_{j}=C \omega^{j}+C^{p} \omega^{-j}$. We may assume that $C \neq \pm A$, for otherwise $\left[x, y_{0}, y_{1}\right]=\left[x, z_{0}, z_{1}\right]$. Moreover, if $h^{m}=\omega^{j}$ then $f_{A}(h)=y_{j}$ and $f_{C}(h)=z_{j}$.

As in the proof of the hyperbolic case, we derive from Proposition 4.2 that

$$
\left(\frac{\left(f_{A}(h)^{2}-4\right)\left(f_{C}(h)^{2}-4\right)}{p}\right)=\left(\frac{\left(f_{A \omega}(h)^{2}-4\right)\left(f_{C \omega}(h)^{2}-4\right)}{p}\right)=-1
$$

cannot hold for any $h \in H$. To be able to use Theorem 4.10, we want to reparametrize (12) as polynomials in $s \in \mathbb{F}_{p}$, using Lemma 4.11. Denote

$$
g_{A}(s) \stackrel{\text { def }}{=}\left(1+s^{2}\right)^{2 m}\left[f_{A}(h(s))^{2}-4\right]
$$

where

$$
h(s)=\frac{2 s+i\left(1-s^{2}\right)}{1+s^{2}}=\frac{-i(s+i)^{2}}{1+s^{2}}=\frac{-i(s+i)}{(s-i)} .
$$

Let also $k_{1}=g_{A} g_{C}$ and $k_{2}=g_{A \omega} g_{C \omega}$. Then (12) is equivalent to

$$
\left(\frac{k_{1}(s)}{p}\right)=\left(\frac{k_{2}(s)}{p}\right)=-1
$$

As in the proof of the hyperbolic case, denote by $N_{(-1,-1)}$ the number of $s \in \mathbb{F}_{p}$ for which (13) holds. Our goal is to get a contradiction by showing that $N_{(-1,-1)}>0$. Note that $g_{A}(s) \neq 0$ for $s \in \mathbb{F}_{p}$ because $g_{A}(s)=\left(1+s^{2}\right)^{2 m}\left(y_{j}^{2}-4\right)$ for some $y_{j}$ as above, and $s \neq \pm i$ and $y_{j} \neq \pm 2$. Thus $k_{i}(s) \neq 0$ neither, and $\left(\frac{k_{i}(s)}{p}\right) \in\{1,-1\}$. As in equations (8)-10 in the hyperbolic case, we get that

$$
N_{(-1,-1)}=\frac{1}{4}\left[p-M_{\{1\}}-M_{\{2\}}+M_{\{1,2\}}\right],
$$

where for $\emptyset \neq B \in\{1,2\}$, we define $M_{B} \stackrel{\text { def }}{=} \sum_{s \in \mathbb{F}_{p}}\left(\frac{\prod_{j \in B} k_{j}(s)}{p}\right)$.

We use Theorem 4.10 to estimate the $M_{B}$ 's. First, we show that $k_{1}, k_{2} \in \mathbb{F}_{p}[x]$. Notice that

$$
h(s)^{-1}=\frac{(s-i)}{-i(s+i)}=\frac{i(s-i)}{(s+i)}
$$


SO

$$
\begin{aligned}
g_{A}(s)= & \left(1+s^{2}\right)^{2 m}\left[f_{A}(h(s))+2\right]\left[f_{A}(h(s))-2\right] \\
= & \left(1+s^{2}\right)^{2 m}\left(A h(s)^{m}+A^{p} h(s)^{-m}+2\right)\left(A h(s)^{m}+A^{p} h(s)^{-m}-2\right) \\
= & \left(A\left[-i(s+i)^{2}\right]^{m}+A^{p}\left[i(s-i)^{2}\right]^{m}+2\left[1+s^{2}\right]^{m}\right) . \\
& \cdot\left(A\left[-i(s+i)^{2}\right]^{m}+A^{p}\left[i(s-i)^{2}\right]^{m}-2\left[1+s^{2}\right]^{m}\right) .
\end{aligned}
$$

The last expression shows that $g_{A}(s) \in \mathbb{F}_{p^{2}}[s]$. Its degree is $4 m$ : indeed, the leading coefficient is

$$
(-1)^{m}\left(A^{2}+A^{2 p}\right)+2 A^{p+1}-4,
$$

and for $m$ even this coefficient equals $\left(A+A^{p}\right)^{2}-4=y_{0}^{2}-4$ which is not zero since $y_{0} \neq \pm 2$ (see Lemma 2.3). For $m$ odd, this coefficient is

$$
-\left(A+A^{p}\right)^{2}+4\left(A^{p+1}-1\right)=-y_{0}^{2}+4\left(A^{p+1}-1\right)
$$

which is not zero because $A^{p+1}-1=\frac{4}{x^{2}-4}$ is not a square in $\mathbb{F}_{p}$ when $x$ is elliptic.

As $\mathbb{F}_{p^{2}}=\mathbb{F}_{p}+i \mathbb{F}_{p}$, we can write $g_{A}=g_{A}^{\prime}+i g_{A}^{\prime \prime}$, where $g_{A}^{\prime}, g_{A}^{\prime \prime} \in \mathbb{F}_{p}[s]$. By definition, for every $s \in \mathbb{F}_{p}$, we have $h=h(s) \in H$, and

$$
g_{A}(s)=\left(1+s^{2}\right)^{2 m}\left[f_{A}(h)^{2}-4\right] \in \mathbb{F}_{p}
$$

so $g_{A}^{\prime \prime}(s)=0$ for every $s \in \mathbb{F}_{p}$. Since $\operatorname{deg}\left(g_{A}^{\prime \prime}\right) \leq 4 m<p$, we conclude that $g_{A}^{\prime \prime}$ is the zero polynomial, hence $g_{A}(s)=g_{A}^{\prime}(s) \in \mathbb{F}_{p}[s]$ and so $k_{1}, k_{2} \in \mathbb{F}_{p}[x]$.

Next, we wish to show that $k_{1}, k_{2}$ and $k_{1} k_{2}$ are not squares in $\overline{\mathbb{F}_{p}}[x]$. There is a one-to-one correspondence between the roots of $g_{A}$ in $\overline{\mathbb{F}_{p}}$ and the roots of

$$
r_{A}(h) \stackrel{\text { def }}{=}\left(A h^{2 m}+2 h^{m}+A^{p}\right)\left(A h^{2 m}-2 h^{m}+A^{p}\right)
$$

in $\overline{\mathbb{F}_{p}}$ given by

$$
\begin{aligned}
\alpha & \mapsto h(\alpha)=\frac{-i(\alpha+i)}{(\alpha-i)} \\
\frac{1+i h}{h+i}=\alpha(h) & \leftrightarrow h,
\end{aligned}
$$

because $\pm i$ is never a root of $g_{A}$ (recall that $g_{A}(s) \in \mathbb{F}_{p}[s]$ has the form from (16)) and $-i$ never a root of $r_{A}$ (because $r_{A}(h)=h^{2 m}\left(f_{A}(h)^{2}-4\right),-i \in H$ and thus $f_{A}(-i)=y_{j}$ for some $y_{j}$ as above, and $y_{j} \neq \pm 2$ ). It is easier to analyze the roots of $r_{A}$ than those of $g_{A}$ : if $h$ is a root of $r_{A}$ then

$$
h^{m}=\frac{ \pm 1 \pm \sqrt{1-\kappa(x)}}{A}
$$

where $\kappa(x)=A^{p+1}=\frac{x^{2}}{x^{2}-4}$. Now note the following:

- The four possible values of $h^{m}$ are distinct and different from zero (this follows from $\kappa(x) \neq 0,1)$.

- Because $(m, p)=1$, the four polynomials $h^{m}-\frac{ \pm 1 \pm \sqrt{1-\kappa(x)}}{A}$ are separable, so $r_{A}$ has $4 m$ distinct roots in $\overline{\mathbb{F}_{p}}$, and so does $g_{A}$. 
- If $A \neq \pm C$, the $4 m$ roots of $r_{A}$ are distinct from the $4 m$ roots of $r_{C}$ : certainly $\frac{1+\sqrt{1-\kappa(x)}}{A} \neq \pm \frac{1+\sqrt{1-\kappa(x)}}{C}$, and if $\frac{1+\sqrt{1-\kappa(x)}}{A}= \pm \frac{1-\sqrt{1-\kappa(x)}}{C}$ we obtain

$$
\begin{gathered}
C= \pm A \cdot \frac{1-\sqrt{1-\kappa(x)}}{1+\sqrt{1-\kappa(x)}} \\
\kappa(x)=C^{p+1}=A^{p+1}\left(\frac{1-\sqrt{1-\kappa(x)}}{1+\sqrt{1-\kappa(x)}}\right)^{p+1}=\kappa(x) \xi^{p+1}
\end{gathered}
$$

with $\xi=\frac{1-\sqrt{1-\kappa(x)}}{1+\sqrt{1-\kappa(x)}} \in \mathbb{F}_{p}$ because $1-\kappa(x)=\frac{-4}{x^{2}-4}$ is a square in $\mathbb{F}_{p}$. Then $\xi= \pm 1$, that is, $C= \pm A-$ a contradiction. Hence $k_{1}=g_{A} g_{C}$ and $k_{2}=g_{A \omega} g_{C \omega}$ are separable of degree $8 m$ each.

- Finally, if $C \neq \pm A$, the polynomial $k_{1} k_{2}=g_{A} g_{A \omega} g_{C} g_{C \omega}$ is not a square in $\overline{\mathbb{F}_{p}}[x]$ : it is separable unless $A= \pm C \omega$ or $A \omega= \pm C$, but the two cannot hold simultaneously.

We can now apply Theorem 4.10 to obtain the same bounds on the $M_{B}$ 's as in the hyperbolic case, and from (14) we now obtain

$$
\begin{array}{rll}
N_{(-1,-1)} & \geq & \frac{1}{4}[p-2(8 m-1) \sqrt{p}-(16 m-1) \sqrt{p}] \\
& = & \frac{1}{4}[p-32 m \sqrt{p}+3 \sqrt{p}] \\
& \stackrel{m=\frac{p+1}{{ }^{d}}}{\geq} & \frac{1}{4}\left[p-\frac{16(p+1)}{d} \sqrt{p}+3 \sqrt{p}\right] \\
d \geq 16 \sqrt{p+1} & \frac{\sqrt{p}}{4}[\sqrt{p}-\sqrt{(p+1)}+3]>0 .
\end{array}
$$

Remark 4.12. As we noted in Remark 4.7 above, it is conceivable that a stronger version of Proposition 4.3 holds. Let us point to the phase in the current argument that fails in this more general setting. The simplest case to consider if that of $x, x^{\prime} \in \mathbb{F}_{p}$ both hyperbolic of maximal order, so $d_{p}(x)=d_{p}\left(x^{\prime}\right)=\frac{p-1}{2}$. Assume that $x=\omega+\omega^{-1}$ and $x^{\prime}=\omega^{\prime}+\omega^{\prime-1}$, and that $\omega^{\prime}=\omega^{r}$. Then, in the notation of Section 4.2.2, if $y_{j}=\alpha s+\beta s^{-1}$, then $y_{j}^{\prime}=\alpha^{\prime} s^{r}+\beta^{\prime} s^{-r}$, and our goal is to show that $\left(\alpha s+\beta s^{-1}\right)$ and $\left(\alpha^{\prime} s^{r}+\beta^{\prime} s^{-r}\right)$ cannot be of the same type (hyperbolic/elliptic) for too many values of $s \in \mathbb{F}_{p}^{*}$. The problem is that $r$ can be of any order, and is generically of order $\geq \sqrt{p}$. For polynomials of such degree Weil's Theorem 4.10 is useless.

\subsection{Deducing Alternating group from primitivity}

Finally, in this section, we show how to deduce that $Q_{p} \geq \operatorname{Alt}\left(Y^{*}(p)\right)$ whenever $Q_{p}$ is primitive. Throughout this section we denote the symmetric group $\operatorname{Sym}(n)$ by $S_{n}$ and Alt $(n)$ by $A_{n}$. Here we use the following result of Guralnick and Magaard, classifying primitive subgroups of $S_{n}$ containing an element with at least $n / 2$ fixed points. This theorem relies heavily on the CFSG. We adjust the statement of the theorem to our needs - the original statement in [GM98] is more detailed. In the statement we use the notation $\operatorname{Soc}(G)$ for the socle of the group $G$ (see Section 5.3 for details), and the standard notation $G_{1} \prec G_{2}$ for the wreath product of two groups. 
Theorem 4.13 ([GM98, Theorem 1]). Let $G \leq \mathrm{S}_{\mathrm{n}}$ be a primitive group, and let $x \in G$ have at least $n / 2$ fixed points. Then one of the following holds:

1. $G=\operatorname{Aff}(2, k)$ is the affine group acting on $\mathbb{F}_{2}{ }^{k}$ and $x$ is a transvection ${ }^{5}$ and is, in particular, an involution. In this case $x$ has exactly $n / 2$ fixed points.

2. There are $r \geq 1, m \geq 5$ and $1 \leq k \leq m / 4$ such that $n=\left(\begin{array}{c}m \\ k\end{array}\right)^{r}$, the group $S_{m}$ acts on the set $\Delta$ of $k$-subsets of $\{1, \ldots, m\}$ in the natural way, $G \leq S_{m} 2 S_{r}$ acts on $\Delta^{r}$ and $\operatorname{Soc}(G)=A_{m}^{r}$.

3. For some $r \geq 1, n=6^{r}$, the group $S_{6}$ acts on $\Delta=\{1, \ldots, 6\}$ by applying an outer automorphism ${ }^{6}, G \leq S_{6}$ \ $S_{r}$ acts on $\Delta^{r}$ and $\operatorname{Soc}(G)=A_{6}{ }^{r}$.

4. The group $G$ is some variant of an orthogonal group over the field of two elements acting on some collection of 1-spaces or hyperplanes, and the element $x$ is an involution.

The following lemma helps us rule out Case 2 of the above theorem with $r=1$.

Lemma 4.14. Consider the embedding $\iota: S_{m} \hookrightarrow S_{n}$ given by the natural action of the symmetric group $S_{m}$ on the set $\Delta$ of $n=\left(\begin{array}{c}m \\ k\end{array}\right) k$-subsets of $m$, for some $2 \leq k \leq \frac{m}{4}$. If, for some $\pi \in S_{m}$, the image $\iota(\pi)$ has a cycle of size divisible by $q$ and a cycle of size divisible by $s$ for some distinct primes $q$ and $s$, then $\iota(\pi)$ also has a cycle of size divisible by qs.

Proof. Assume that $\left\{a_{1}, \ldots, a_{k}\right\} \in \Delta$ belongs to a cycle $\alpha$ of length divisible by $q$ in $\iota(\pi)$. Assume that in $\pi$, the elements $a_{1}, \ldots, a_{k}$ belong to $t$ distinct cycles: the elements $a_{1}, \ldots, a_{\ell_{1}}$ belong to the cycle $\sigma_{1}$, the elements $a_{\ell_{1}+1}, \ldots, a_{\ell_{2}}$ belong to the cycle $\sigma_{2}$, and so on (each $\sigma_{j}$ may contain additional elements not from $\left.\left\{a_{1}, \ldots, a_{k}\right\}\right)$. Let $o_{1}$ be the smallest power of $\sigma_{1}$ that maps $\left\{a_{1}, \ldots, a_{\ell_{1}}\right\}$ to itself. Define $o_{2}, \ldots, o_{t}$ analogously. Then, $q \mid \operatorname{lcm}\left(o_{1}, \ldots, o_{t}\right)$. In particular, $q \mid o_{i}$ for some $i$, and so $q|| \sigma_{i} \mid$. Without loss of generality, assume $q|| \sigma_{1} \mid$, so that $a_{1}$ belongs to a cycle $\sigma=\sigma_{1}$ of $\pi$ of size divisible by $q$. Likewise, assume that $b_{1}$ belongs to a cycle $\tau$ of $\pi$ of size divisible by $s$.

Denote $A=\{1, \ldots, m\} \backslash(\sigma \cup \tau)$ (namely, $A$ consists of the elements not belonging to the cycle $\sigma$ nor to $\tau)$. Assume first that $\sigma \neq \tau$. If $|A| \geq k-2$, then a $k$-subset containing $a_{1}, b_{1}$ and $k-2$ elements from $A$ belongs to a cycle of $\iota(\pi)$ of size divisible by qs. If $|A|<k-2$, then, as $k \leq \frac{m}{4}$, at least one of $\sigma$ or $\tau$ has more than $k$ element. Assume without loss of generality it is $\sigma$. Consider the $k$-subset $\left\{b_{1}, a_{1}, \pi\left(a_{1}\right), \pi^{2}\left(a_{1}\right), \ldots, \pi^{k-2}\left(a_{1}\right)\right\}$. This subset belongs to a cycle of $\iota(\pi)$ of size $\operatorname{lcm}(|\tau|,|\sigma|)$, which, in particular, is a multiple of $q s$.

Finally, assume $\sigma=\tau$. Then $q s|| \sigma \mid$. If the length of $\sigma$ is at least $k+1$, the $k$-subset $\left\{a_{1}, \pi\left(a_{1}\right), \pi^{2}\left(a_{1}\right), \ldots, \pi^{k-2}\left(a_{1}\right), \pi^{k-1}\left(a_{1}\right)\right\}$ belongs to a cycle of $\iota(\pi)$ of size dividing $q s$. If $|\sigma| \leq k$ then $A$ contains more than $k-1$ elements, and the $k$-subset containing $a_{1}$ and $k-1$ elements from $A$ belongs to a cycle of $\iota(\pi)$ of size dividing $q s$.

Proposition 4.15. Let $p \equiv 3(4)$ be prime. If $Q_{p}$ is primitive, then $Q_{p} \geq \operatorname{Alt}\left(Y^{*}(p)\right)$.

Proof. Consider $\operatorname{rot}_{1} \in Q_{p}$. Among the $\frac{p(p-3)}{4}$ elements in $Y^{*}(p), \frac{(p-1)(p-3)}{8}$ belong to cycles of length at least 3 and dividing $\frac{p-1}{2}$, and $\frac{(p+1)(p-3)}{8}$ belong to cycles of length at least 3 and dividing $\frac{p+1}{2}$ (see Table 2). Since $\operatorname{gcd}\left(\frac{p-1}{2}, \frac{p+1}{2}\right)=1$, the permutation $\sigma=\operatorname{rot}_{1}^{(p+1) / 2}$ fixes

\footnotetext{
${ }^{5}$ To be sure, $x$ is a transvection when $\operatorname{Aff}(2, k)$ is embedded in GL $(2, k+1)$ as the matrices with bottom row $(0, \ldots, 0,1)$.

${ }^{6}$ Namely, for some fixed $\varphi \in \operatorname{Aut}\left(S_{6}\right) \backslash \operatorname{Inn}\left(S_{6}\right)$, the permutation $\sigma \in S_{6}$ acts on $\Delta$ by $\sigma . i=\varphi(\sigma)(i)$.
} 
exactly $\frac{(p+1)(p-3)}{8}>\frac{\left|Y^{*}(p)\right|}{2}$ elements of $Y^{*}(p)$. Thus $Q_{p}$ satisfies the assumptions in Theorem 4.13. We can now rule out all options except for $Q_{p}=\operatorname{Alt}\left(Y^{*}(p)\right)$ or $Q_{p}=\operatorname{Sym}\left(Y^{*}(p)\right)$.

Cases 1 and 4 are immediately ruled out because the permutation $\sigma \in Q_{p}$ is not an

involution. Case 2 with $r \geq 2$ and Case 3 are immediately ruled out because $\left|Y^{*}(p)\right|=\frac{p(p-3)}{4}$ is not a proper power nor equal to six. It remains to consider Case 2 with $r=1$.

Let $q$ be some prime factor of $\frac{p-1}{2}$, and let $s$ be some prime factor of $\frac{p+1}{2}$. By Table 2 , $\operatorname{rot}_{1}$ contains cycles of size divisible by $q$ (indeed, even of size $q$ exactly), and of size divisible by $s$. However, it does not contain any cycle of size divisible by $q s$. Using Lemma 4.14, this rules out Case 2 from Theorem 4.13 with $r=1$ and $k \geq 2$. The remaining case, that of Case 2 with $r=k=1$, is precisely the case that the group in question is either $A_{n}$ or $S_{n}$.

This finishes the proofs of Theorem 1.4 and of Corollary 1.5: Theorem 1.4 is now a consequence of Theorem 4.1 and Proposition 4.15, while Corollary 1.5 follows from Corollary 4.6 and Proposition 4.15 .

\section{Strong Approximation for Square Free Composite Moduli}

In this section we derive our main application of the results on the groups $Q_{p}$ and show that $\Gamma$ acts transitively on $X^{*}(n)$ for various square-free composite values $n=p_{1} \cdots p_{k}$. First, in Section 5.1, we prove that if $Q_{p_{j}} \geq \operatorname{Alt}\left(Y^{*}\left(p_{j}\right)\right)$ for every $j=1, \ldots, k$, then $\Gamma$ acts transitively on $Y^{*}(n)$. In Section 5.2 we strengthen this result to showing that, moreover, $\Gamma$ acts transitively on $X^{*}(n)$, namely, that strong approximation for the Markoff equation holds in modulo $n$, thus proving Theorem 1.6.

At this point, we are able to prove Theorem 1.4 that $Q_{p} \geq \operatorname{Alt}\left(Y^{*}(p)\right)$ for $p \equiv 3(4)$ satisfying the assumptions in the statement of Theorem 1.4, only while relying on the classification of finite simple groups (CFSG) - see Section 4.3. However, the CFSG is not necessary for establishing the transitivity of $\Gamma$ on $X^{*}(n)$ when $n=p_{1} \cdots p_{k}$ and $p_{1}, \ldots, p_{k}$ are distinct primes satisfying the assumptions in Theorems 1.3 or 1.4 (this is Corollary 1.7). In Section 5.3 we give an alternative proof for the transitivity of $\Gamma$ on $X^{*}(n)$, which uses only the primitivity of $Q_{p}$, as in Theorem 4.1, thus proving Theorem 1.9. The point is that we want to provide a proof of the transitivity on $X^{*}(n)$ which can be potentially understood in full, from basic principles, by a motivated reader. This is practically impossible if one relies on the CFSG.

\subsection{Transitivity of $\Gamma$ on $Y^{*}(n)$}

Here we prove the following lemma:

Lemma 5.1. Let $n=p_{1} \cdots p_{k}$ be a product of distinct primes. If $Q_{p_{j}} \geq \operatorname{Alt}\left(Y^{*}\left(p_{j}\right)\right)$ for $j=1, \ldots, k$, then $\Gamma$ acts transitively on $Y^{*}(n)$.

Moreover, $Q_{n}$, which is a subgroup of $\operatorname{Sym}\left(Y^{*}\left(p_{1}\right)\right) \times \ldots \times \operatorname{Sym}\left(Y^{*}\left(p_{k}\right)\right)$, contains $\operatorname{Alt}\left(Y^{*}\left(p_{1}\right)\right) \times$ $\ldots \times \operatorname{Alt}\left(Y^{*}\left(p_{k}\right)\right)$.

Proof. We prove the proposition by induction on $k$, the case $k=1$ being trivial. Assume $k \geq 2$. It is enough to show that for every $j=1, \ldots, k$,

$$
Q_{n} \geq 1 \times \ldots \times 1 \times \operatorname{Alt}\left(Y^{*}\left(p_{j}\right)\right) \times 1 \times \ldots \times 1 .
$$

Recall that $Y^{*}(3)=\emptyset$, so we may assume $3 \nmid n$. Without loss of generality we assume that $j=k$. We first prove (17) assuming $p_{k} \geq 5$. Note that $\left|Y^{*}\left(p_{k}\right)\right| \geq 5$ (see Lemmas 2.2 and 
2.3), and so Alt $\left(Y^{*}\left(p_{k}\right)\right)$ is simple. This group is never a composition (Jordan-Hölder) factor of $\operatorname{Alt}\left(Y^{*}\left(p_{\ell}\right)\right)$ when $p_{k} \neq p_{\ell}$, because ${ }^{7}\left|Y^{*}\left(p_{k}\right)\right| \neq\left|Y^{*}\left(p_{\ell}\right)\right|$. Now consider the normal series

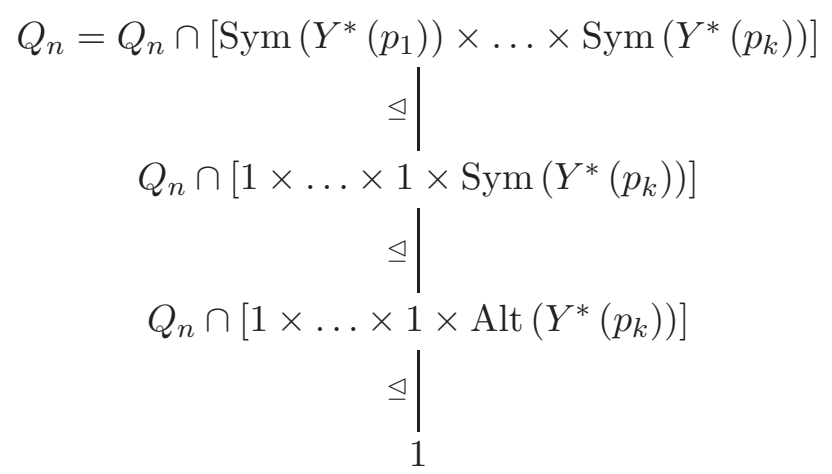

The group $Q_{p_{k}}$ is a quotient of $Q_{n}$, and so $\operatorname{Alt}\left(Y^{*}\left(p_{k}\right)\right)$ a composition factor of $Q_{n}$, and thus a composition factor of one of the quotients in (18). But the upper quotient is isomorphic to $Q_{p_{1} \cdots p_{k-1}}$, which by the induction hypothesis has composition factors $\operatorname{Alt}\left(Y^{*}\left(p_{\ell}\right)\right)$ for $\ell \neq k, p_{\ell} \neq 2$ and possibly some copies of $\mathbb{Z} / 2 \mathbb{Z}$ coming from $\operatorname{Sym}\left(Y^{*}\left(p_{\ell}\right)\right) / \operatorname{Alt}\left(Y^{*}\left(p_{\ell}\right)\right)$ or copies of $\mathbb{Z} / 2 \mathbb{Z}$ and $\mathbb{Z} / 3 \mathbb{Z}$ coming from $\operatorname{Sym}\left(Y^{*}(2)\right)$. The middle quotient is either trivial or $\mathbb{Z} / 2 \mathbb{Z}$. Thus Alt $\left(Y^{*}\left(p_{k}\right)\right)$ must be a composition factor of the bottom quotient, so $1 \times \ldots \times 1 \times$ $\operatorname{Alt}\left(Y^{*}\left(p_{k}\right)\right) \leq Q_{n}$.

Finally, if $p_{k}=2$, note that $\left|Y^{*}(2)\right|=4$. The composition factors of Alt (4) are one copy of $\mathbb{Z} / 3 \mathbb{Z}$ and two copies of $\mathbb{Z} / 2 \mathbb{Z}$. By an argument as above, the factor $\mathbb{Z} / 3 \mathbb{Z}$ must belong to the bottom quotient in (18). Denote

$$
H \stackrel{\text { def }}{=} Q_{n} \cap\left[1 \times \ldots \times 1 \times \operatorname{Alt}\left(Y^{*}(2)\right)\right]=1 \times \ldots \times 1 \times H^{\prime} .
$$

It is easy to check that $H \unlhd Q_{n}$. For every $g_{k} \in \operatorname{Alt}\left(Y^{*}(2)\right)$ there are $g_{1}, \ldots, g_{k-1}$ with $g_{j} \in \operatorname{Sym}\left(Y^{*}\left(p_{j}\right)\right)$ such that $\left(g_{1}, \ldots, g_{k}\right) \in Q_{n}$, thus $H^{\prime} \unlhd \operatorname{Alt}\left(Y^{*}(2)\right) \cong \operatorname{Alt}(4)$. But the only normal subgroup of Alt (4) containing the composition factor $\mathbb{Z} / 3 \mathbb{Z}$ is Alt (4) itself.

\subsection{Transitivity of $\Gamma$ on $X^{*}(n)$}

We now finish the proof of Theorem 1.6 and prove that if $n=p_{1} \cdots p_{k}$ is a product of distinct primes with $Q_{p_{j}} \geq \operatorname{Alt}\left(Y^{*}\left(p_{j}\right)\right)$ for every $1 \leq j \leq k$, then $\Gamma$ acts transitively on $X^{*}(n)$.

We want the proof of this section to work in a slightly greater generality than the assumption that $Q_{p_{j}} \geq \operatorname{Alt}\left(Y^{*}\left(p_{j}\right)\right)$, so that it applies also for the next section, where we do not rely on the CFSG. This is part of the motivation for the following notation:

Notation 5.2. Let $n=p_{1} \cdots p_{k}$ be a product of distinct primes for which $Q_{p_{j}}$ is primitive. We assume further that

- The primes are ordered by the order of the rotations $\operatorname{rot}_{i}$ in the groups $Q_{p_{j}}$, which is

$$
\left|\operatorname{rot}_{1}\right| \text { in } Q_{p}= \begin{cases}3 & p=2 \\ \frac{p\left(p^{2}-1\right)}{4} & p \equiv 1(4) \\ \frac{p^{2}-1}{4} & p \equiv 3(4)\end{cases}
$$

For instance, 7 comes before 5 . We break potential ties by putting the larger prime first: for example, we put 11 before 5 .

\footnotetext{
${ }^{7}$ For $p$ odd the size of $\left|Y^{*}(p)\right|$ is $\frac{p(p \pm 3)}{4}$ as given in Section 2 , and $\left|Y^{*}(2)\right|=4$.
} 
- Without loss of generality, $2,5,7,11 \mid n$ and so the first four primes are $2,7,11,5$ (in that order). This assumption is possible because in these four cases, computer simulations indicate that $Q_{p}=\operatorname{Sym}\left(Y^{*}(p)\right)$ is the full symmetric group, so our assumptions always hold.

Furthermore, for $j=1, \ldots, k$,

- Let $M_{j}=p_{1} \cdots p_{j}$ denote the product of the first $j$ primes.

- Let $\Omega_{j} \unlhd \Gamma$ denote the kernel of the action of $\Gamma$ on $Y^{*}\left(M_{j}\right)$. Note than $\Omega_{j+1} \unlhd \Omega_{j}$. $\quad \Omega_{j}$

- Let $\Lambda_{j} \unlhd \Gamma$ denote the kernel of the action of $\Gamma$ on $X^{*}\left(M_{j}\right)$. Note that $\Lambda_{j+1} \unlhd \Lambda_{j} \unlhd \Omega_{j}$. $\Lambda_{j}$

Finally, for every prime $p$, we let $\pi_{p}: \Gamma \rightarrow Q_{p}$ denote the projection.

In Section 5.3 we shall prove the following lemma without relying on the CFSG:

Lemma 5.3. Let $n=p_{1} \cdots p_{k}$ with $Q_{p_{j}}$ primitive for $j=1, \ldots, k$ as in Notation 5.2. Then, for every $j=2, \ldots, k$, the image of $\Omega_{j-1}$ in $Q_{p_{j}}$ contains a subgroup $H_{j} \leq \operatorname{Sym}\left(Y^{*}\left(p_{j}\right)\right)$ satisfying:

1. $H_{j}$ is transitive on $Y^{*}\left(p_{j}\right)$

2. $H_{j}$ is isomorphic to a direct product of non-abelian simple groups $8 T_{1} \times \ldots \times T_{m}$ for some $m=m(j) \in \mathbb{Z}_{\geq 1}$.

In particular, $\Omega_{j-1}$ acts transitively on $Y^{*}\left(p_{j}\right)$ and $\Gamma$ acts transitively on $Y^{*}(n)$.

Note that if we assume that $Q_{p_{j}} \geq \operatorname{Alt}\left(Y^{*}\left(p_{j}\right)\right)$, the conclusion of Lemma 5.3 follows immediately from Lemma 5.1: indeed, for $p \geq 5$, Alt $\left(Y^{*}(p)\right)$ is indeed transitive on $Y^{*}(p)$ and is a product of a single non-abelian simple group. So Lemma 5.3 is already proven relying on the CFSG, or if one assumes that $p_{j} \equiv 1$ (4) for $j=1, \ldots, k$. In the remaining part of this subsection we rely only on the conclusion of Lemma 5.3. We assume Notation 5.2 throughout.

Lemma 5.4. For $j=2, \ldots, k$, the group $\Lambda_{j-1}$ acts transitively on $Y^{*}\left(p_{j}\right)$.

Proof. Consider the normal series

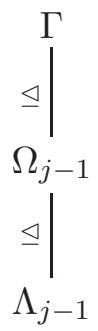

and its projection on $Q_{p_{j}}$ via $\pi_{j}: \Gamma \rightarrow Q_{p_{j}}$. By Lemma 5.3, $\pi_{j}\left(\Omega_{j-1}\right) \geq H_{j}$ where $H_{j}$ acts transitively on $Y^{*}\left(p_{j}\right)$ and is a direct product of non-abelian simple groups. As $\Omega_{j-1}$ fixes $Y^{*}\left(M_{j-1}\right)=Y^{*}\left(p_{1}\right) \times \ldots \times Y^{*}\left(p_{j-1}\right)$, its action on $X^{*}\left(M_{j-1}\right)$ fixes every 4-block and only permutes elements inside the 4-blocks, hence the image of $\Omega_{j-1}$ in $\Gamma_{M_{j-1}}$ is a subgroup of $\operatorname{Sym}(4)^{\left|Y^{*}\left(p_{1}\right)\right|+\ldots+\left|Y^{*}\left(p_{j-1}\right)\right|}$. Hence this image is solvable of order $2^{\alpha} \cdot 3^{\beta}$ for some $\alpha, \beta \in \mathbb{Z}_{\geq 0}$, so all its composition factors are either $\mathbb{Z} / 2 \mathbb{Z}$ or $\mathbb{Z} / 3 Z$. We deduce that the quotient $\Omega_{j-1} / \Lambda_{j-1}$ has only composition factors $\mathbb{Z} / 2 \mathbb{Z}$ and $\backslash$ or $\mathbb{Z} / 3 \mathbb{Z}$. Let

$$
\Omega_{j-1}=N_{0} \unrhd N_{1} \unrhd \ldots \unrhd N_{r}=\Lambda_{j-1}
$$

\footnotetext{
${ }^{8}$ Note that we assume $j \geq 2$. Indeed, this does not hold for $p_{1}=2$ : there are no simple non-abelian subgroups inside $\operatorname{Sym}\left(Y^{*}(2)\right) \cong \operatorname{Sym}(4)$.
} 
be a normal series with quotients $\mathbb{Z} / 2 \mathbb{Z}$ and $\backslash$ or $\mathbb{Z} / 3 \mathbb{Z}$. Note that the index $\left[H_{j}: \pi_{j}\left(N_{1}\right) \cap H_{j}\right]$ is at most 3 , but as $H_{j}$ is a direct product of non-abelian simple groups, it has no proper subgroups of index ${ }^{9} \leq 3$, hence $\pi_{j}\left(N_{1}\right) \geq H_{j}$. By induction, the same argument shows that $\pi_{j}\left(N_{\ell}\right) \geq H_{j}$ for every $\ell$, and, in particular, $\pi_{j}\left(\Lambda_{j-1}\right) \geq H_{j}$.

Lemma 5.5. For $j=5, \ldots, k$ (so $p_{j} \geq 13$ ), $\Lambda_{j-1}$ acts transitively on $X^{*}\left(p_{j}\right)$.

Proof. Our strategy is to find a triple $(x, y, z) \in X^{*}\left(p_{j}\right)$ and elements in $\Lambda_{j-1}$ mapping $(x, y, z)$ to the other elements in its 4-block: $(x,-y,-z),(-x, y,-z)$ and $(-x,-y, z)$. Together with the transitivity of $\Lambda_{j-1}$ on $Y^{*}\left(p_{k}\right)$ established in Lemma 5.4, this would complete the proof. As in other places in this paper, we deal separately with the case $p_{j} \equiv 1(4)$ and the case $p_{j} \equiv 3(4)$, the argument in the former case being simpler.

Case 1: $p=p_{j} \equiv 1(4)$

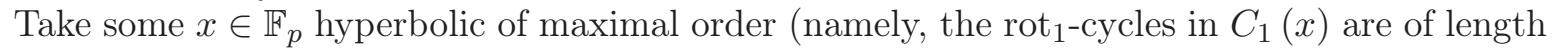
$p-1 \geq 12$ each). Since 0 has order $4, x \neq 0$ and $(0, x, i x) \in X^{*}(p)$. Let $(r, s, t) \in X^{*}(p)$ be another solution with $r$ elliptic. As all $\operatorname{rot}_{1}$-cycles in $C_{1}(0)$ have length 4 and $(p+1 \equiv 2 \bmod 4)$, we get that $\operatorname{rot}_{1}^{p+1}$ fixes all four elements in $[r, s, t]$ while mapping $(0, x, i x) \mapsto(0,-x,-i x)$. By Lemma 5.4, there is some $g \in \Lambda_{j-1}$ mapping $[0, x, i x] \mapsto[r, s, t]$. The element $h_{1}=$ $g^{-1} \cdot \operatorname{rot}_{1}{ }^{-(p+1)} \cdot g \cdot \operatorname{rot}_{1}^{p+1}$ is in $\Lambda_{j-1}\left(\right.$ as $\left.\Lambda_{j-1} \unlhd \Gamma\right)$ and maps $(0, x, i x) \mapsto(0,-x,-i x)$.

Since $x$ is maximal hyperbolic, its order is $(p-1)$ which is divisible by 4 . Hence $-x$ is also maximal hyperbolic. Let now $\left(r^{\prime}, s^{\prime}, t^{\prime}\right) \in X^{*}(p)$ be a solution with $s^{\prime}$ elliptic. Note that $\left(\frac{p^{2}-1}{4} \equiv 0 \bmod p+1\right)$ while $\left(\frac{p^{2}-1}{4} \equiv \frac{p-1}{2} \bmod p-1\right)$. Thus $\operatorname{rot}_{2}{ }^{\left(p^{2}-1\right) / 4}$ fixes all four elements in $\left[r^{\prime}, s^{\prime}, t^{\prime}\right]$ while mapping $(0, x, i x) \mapsto(0, x,-i x)$ and $(0,-x,-i x) \mapsto(0,-x, i x)$. By Lemma 5.4, there is some $g^{\prime} \in \Lambda_{j-1}$ mapping $[0, x, i x] \mapsto\left[r^{\prime}, s^{\prime}, t^{\prime}\right]$. The element $h_{1}=\left(g^{\prime}\right)^{-1}$. $\operatorname{rot}_{1}{ }^{-\left(p^{2}-1\right) / 4} \cdot g^{\prime} \cdot \operatorname{rot}_{1}{ }^{\left(p^{2}-1\right) / 4}$ is in $\Lambda_{j-1}$ and maps $(0, x, i x) \mapsto(0, x,-i x)$ and $(0,-x,-i x) \mapsto$ $(0,-x, i x)$.

Case 2: $p=p_{j} \equiv 3(4)$

In Proposition 5.6 below, we prove there is a solution $(x, y, z) \in X^{*}(p)$ with both $x$ and $y$ elliptic of order divisible by 4 . In this case, $-x$ has the same order as $x$, say this order is $4 m$ and note that $4 m \mid(p+1)$. Let $(r, s, t) \in X^{*}(p)$ be another solution with $r$ hyperbolic. As $p-1 \equiv 2(4)$, there is a number $q$ with $(q \equiv 2 m \bmod 4 m)$ and $(q \equiv 0 \bmod p-1)$. We get that $\operatorname{rot}_{1}{ }^{q}$ fixes all four elements in $[r, s, t]$ while mapping $(x, y, z) \mapsto(x,-y,-z)$ and $(-x,-y, z) \mapsto(-x, y,-z)$. By Lemma 5.4, there is some $g \in \Lambda_{j-1}$ mapping $[x, y, z] \mapsto$ $[r, s, t]$. The element $h_{1}=g^{-1} \cdot \operatorname{rot}_{1}{ }^{-q} \cdot g \cdot \operatorname{rot}_{1}^{q}$ is in $\Lambda_{j-1}$ and maps $(x, y, z) \mapsto(x,-y,-z)$ and $(-x,-y, z) \mapsto(-x, y,-z)$. In the same fashion, we find an element of $\Lambda_{j-1}$ mapping $(x, y, z) \mapsto(-x, y,-z)$ and we are done.

Modulo Proposition 5.6 which we prove at the end of this subsection, we can now complete the proofs of Theorem 1.6 and Corollary 1.7:

Proof of Theorem 1.6. We use Notation 5.2. We need to show that $\Gamma$ acts transitively on $X^{*}(n)$. We prove that $\Gamma$ acts transitively on $X^{*}\left(M_{j}\right)$ for $j=1, \ldots, k$ (recall that $M_{k}=n$ ). For $j=4$ we verified by computer that $\Gamma$ is transitive on $X^{*}(2 \cdot 5 \cdot 7 \cdot 11)$. For $j \geq 5$, we use

\footnotetext{
${ }^{9}$ To be sure, the reason that $H=T_{1} \times \ldots \times T_{m}$ with $T_{1}, \ldots, T_{m}$ finite non-abelian simple groups has no subgroups of index 2 or 3 is that the normal subgroups of $H$ are $B_{1} \times \ldots \times B_{m}$ where $B_{i} \in\left\{1, T_{i}\right\}$ for every $i$ (this is standard: if $N \unlhd H$ and $N \cap T_{1} \neq 1$, then $1 \neq\left[N, T_{1}\right] \unlhd T_{1}$, and so $\left[N, T_{1}\right]=T_{1}$ and $N \geq T_{1}$ ). In particular, since the smallest non-abelian simple group is Alt (5), any proper normal subgroup of $H$ is of index at least 60 . If $K \leq H$ has index 2 or 3 , then its core, $\cap_{h \in H} h K h^{-1}$, is proper normal subgroup of index at most 6 , which is impossible.
} 
induction and assume that $\Gamma$ acts transitively on $X^{*}\left(M_{j-1}\right)$. From Lemma 5.5 it follows that $\Gamma$ is transitive on $X^{*}\left(M_{j}\right)$.

We complete the subsection with the proposition we use in the proof of case 2 in Lemma 5.5:

Proposition 5.6. For every prime $p \neq 3,11$ with $p \equiv 3(4)$, there is a solution $(x, y, z) \in$ $X^{*}(p)$ with two coordinates elliptic of order divisible by 4.

In the proof of Proposition 5.6 we use notation as in Section 4.2.3. As $4 \mid(p+1)$, if $\omega \in H$ is not a square then 4||$\omega \mid$. Thus, it is enough to find a solution $(x, y, z) \in X^{*}(p)$ with $x, y$ elliptic and the corresponding $\omega_{x}, \omega_{y}$ not squares in $H$.

Lemma 5.7. Assume $y=\omega+\omega^{-1}$ is elliptic (so $\omega \in H$ ). Then $\omega$ is a square in $H$ if and only if $y+2$ is a square in $\mathbb{F}_{p}$.

Proof. Note that $y+2=\omega+\omega^{-1}+2=\left(\omega^{1 / 2}+\omega^{-1 / 2}\right)^{2}$. If $\omega^{1 / 2} \in H$ then $\omega^{1 / 2}+\omega^{-1 / 2} \in \mathbb{F}_{p}$. On the other hand, if $\omega^{1 / 2} \notin H$, then $\omega^{(p+1) / 2}=-1$ and so $\omega^{1 / 2}+\omega^{-1 / 2} \notin \mathbb{F}_{p}$, because

$$
\left(\omega^{1 / 2}+\omega^{-1 / 2}\right)^{p}=\omega^{(p+1) / 2} \omega^{-1 / 2}+\omega^{-(p+1) / 2} \omega^{1 / 2}=-\left(\omega^{-1 / 2}+\omega^{1 / 2}\right) \neq\left(\omega^{1 / 2}+\omega^{-1 / 2}\right)
$$

(the last inequality stems from $\left.\left(\omega^{1 / 2}+\omega^{-1 / 2}\right)^{2}=y+2 \neq 0\right)$.

Proof of Proposition 5.6. Fix $x \in \mathbb{F}_{p}$ elliptic of maximal order $(p+1)$. So 4||$\omega_{x} \mid=p+1$. By Lemma 5.7, it is enough to find $y, z \in \mathbb{F}_{p}$ such that $(x, y, z) \in X^{*}(p)$ is a solution, $y$ is elliptic and $y+2$ is a non-square. Since $y$ elliptic means that $y^{2}-4=(y+2)(y-2)$ is not a square, we need to find $y, z$ with $(x, y, z) \in X^{*}(p)$ and $y+2$ a non-square and $y-2$ a square.

Imitating the notation from Section 4.2.3, assume $x=\omega+\omega^{-1}$ with $\omega \in H$, choose some $A \in \mathbb{F}_{p^{2}}$ for which $A^{p+1}=\frac{x^{2}}{x^{2}-4}$, and let $f_{A}(h)=A h+A^{p} h^{-1}$ for $h \in H$. Then,

$$
\left\{\left(f_{A}(h), f_{A \omega}(h)\right) \mid h \in H\right\}=\left\{(y, z) \mid(x, y, z) \in X^{*}(p)\right\} .
$$

Recall the parametrization of $H \backslash\{-i\}$ by elements from $\mathbb{F}_{p}$ described in Lemma 4.11: $h(s)=$ $\frac{2 s+i\left(1-s^{2}\right)}{1+s^{2}}=\frac{-i(s+i)}{s-i}$. Define $g_{1}, g_{2} \in \mathbb{F}_{p}[s]$ as follows:

$$
\begin{aligned}
& g_{1}(s) \stackrel{\text { def }}{=}\left(1+s^{2}\right)^{2}\left[f_{A}(h(s))+2\right]=\left(1+s^{2}\right)\left[2 s\left(A+A^{p}\right)+\left(1-s^{2}\right) i\left(A-A^{p}\right)+2\left(1+s^{2}\right)\right] \\
& g_{2}(s) \stackrel{\text { def }}{=}\left(1+s^{2}\right)^{2}\left[f_{A}(h(s))-2\right]=\left(1+s^{2}\right)\left[2 s\left(A+A^{p}\right)+\left(1-s^{2}\right) i\left(A-A^{p}\right)-2\left(1+s^{2}\right)\right] .
\end{aligned}
$$

It is not hard to see that $g_{j}(s) \in \mathbb{F}_{p}[s]$ : indeed, $A+A^{p}, i\left(A-A^{p}\right) \in \mathbb{F}_{p}$. We now show that for large enough $p$, there is some $s \in \mathbb{F}_{p}$ for which

$$
\left(\frac{g_{1}(s)}{p}\right)=-1 \text { and }\left(\frac{g_{2}(s)}{p}\right)=1 .
$$

Denote by $N_{(-1,1)}$ the number of $s \in \mathbb{F}_{p}$ for which (21) holds. Our goal is to show that for large enough $p, N_{(-1,1)}>0$. As in the proof of Proposition 4.3, $g_{1}$ and $g_{2}$ have no zeros inside $\mathbb{F}_{p}$ because there are no solutions in $X^{*}(p)$ involving \pm 2 . So

$$
N_{(-1,1)}=\frac{1}{4} \sum_{s \in \mathbb{F}_{p}}\left(1-\left(\frac{g_{1}(s)}{p}\right)\right)\left(1+\left(\frac{g_{2}(s)}{p}\right)\right) .
$$


For $\emptyset \neq B \subseteq\{1,2\}$, let $M_{B} \stackrel{\text { def }}{=} \sum_{s \in \mathbb{F}_{p}}\left(\frac{\prod_{j \in B} g_{j}(s)}{p}\right)$ and then (22) becomes

$$
N_{(-1,1)}=\frac{1}{4}\left(p-M_{\{1\}}+M_{\{2\}}-M_{\{1,2\}}\right) .
$$

Note that

$$
g_{1}(s) g_{2}(s)=\left(1+s^{2}\right)^{4}\left[f_{A}(h(s))^{2}-4\right]=\left(1+s^{2}\right)^{2} g_{A}(s)
$$

where $g_{A}(s)$ is defined as in Equation (15) in Section 4.2.3 for $m=1$. As our analysis in Section 4.2.3 shows, all roots of $g_{A}$, except for $\pm i$, have multiplicity 1 . Thus, none of $g_{1}$, $g_{2}$ or $g_{1} g_{2}$ is a square in $\overline{\mathbb{F}_{p}}[x]$. Now $g_{1}$ and $g_{2}$ have each at most 4 distinct roots and by Theorem 4.10, $\left|M_{\{1\}}\right|,\left|M_{\lfloor 2\rfloor}\right| \leq 3 \sqrt{p}$. Their product $g_{1} g_{2}$ has at most 6 distinct roots, hence by Theorem $4.10\left|M_{\{1,2\}}\right| \leq 5 \sqrt{p}$. From (23) we get

$$
N_{(-1,1)} \geq \frac{1}{4}(p-2 \cdot 3 \sqrt{p}-5 \sqrt{p})=\frac{p-11 \sqrt{p}}{4} .
$$

So for $p>11^{2}=121$ we have $N_{(-1,1)}>0$ and we are done.

For all primes $p$ with $p \equiv 3(4), p \leq 121$ and $p \neq 3,11$, we verified by a computer there is a solution $(x, y, z) \in X^{*}(p)$ with $x, y$ elliptic and of order divisible by 4 . For example, one can take $(3,3,3) \in X^{*}(7),(6,6,8) \in X^{*}(19),(3,3,3) \in X^{*}(23)$ and $(4,4,9) \in X^{*}(31)$.

\subsection{Transitivity without the classification}

In this section we prove Theorem 1.9 concerning the transitivity of $\Gamma$ in square free composite moduli without relying on the CFSG. We are going to use some strong results from the theory of permutation groups, mostly revolving around O'Nan-Scott theorem. While strong, the proofs of these results are completely contained in the book [DM96] and are not more than a few-page-long each. We stress that if all primes in the decomposition of $n$ are 2 or $(1 \bmod 4)$, then already the proof in the previous sections does not rely on the CFSG.

More concretely, let $n=p_{1} \cdots p_{k}$ be a product of distinct primes, and we assume that $Q_{p_{j}}$ is a primitive permutation group in its action on $Y^{*}\left(p_{j}\right)$ for every $j=1, \ldots, k$. Our goal is to show then that $\Gamma$ acts transitively on $X^{*}(n)$. It is enough to prove Lemma 5.3 above, as we already showed in Section 5.2 how it yields the conclusion we seek. Throughout this subsection we assume Notation 5.2.

The CFSG-free proof of Lemma 5.3 uses the important concept of the socle:

Definition 5.8. A minimal normal subgroup of a non-trivial group $G$ is a normal subgroup $K \neq 1$ of $G$ which does not contain properly any other non-trivial normal subgroup of $G$. The socle of $G$, denoted $\operatorname{Soc}(G)$, is the subgroup generated by the set of all minimal normal subgroups of $G$. Note that $\operatorname{Soc}(G)$ is generated by normal subgroups of $G$ and thus $\operatorname{Soc}(G) \preccurlyeq$ $G$.

For example, if $m \geq 5$ then $\operatorname{Soc}(\operatorname{Sym}(m))=\operatorname{Soc}(\operatorname{Alt}(m))=\operatorname{Alt}(m)$. In contrast, $\operatorname{Soc}(\operatorname{Sym}(4))=\operatorname{Soc}(\operatorname{Alt}(4))=\{1,(12)(34),(13)(24),(14)(23)\}$.

Theorem 5.9 (See [DM96, Theorems 4.3B, Corollary 4.3B and Theorem 4.7A]). Let $G \leq$ Sym $(n)$ be a primitive subgroup. Then exactly one of the following holds:

1. For some prime $p$ and some integer $d$, the group $G$ is permutation isomorphic ${ }^{10}$ to a subgroup of the affine group $\mathrm{Aff}(p, d)$ acting on $\mathbb{F}_{p}^{d}$, so, in particular, $n=p^{d}$. In this

\footnotetext{
${ }^{10}$ Two permutation groups are permutation isomorphic if they are the same permutation groups except for, possibly, the labeling of the points in the sets they act on.
} 
case, $\operatorname{Soc}(G)$ is a regular ${ }^{11}$ elementary abelian subgroup of order $p^{d}$.

2. Soc $(G)=K_{1} \times K_{2}$ where $K_{1}, K_{2} \leqslant G$ are minimal normal subgroups of $G$, which are regular, non-abelian and permutation isomorphic to each other. Moreover ${ }^{12}, C_{G}\left(K_{1}\right)=$ $K_{2}$ and $C_{G}\left(K_{2}\right)=K_{1}$. In addition, $K_{1} \cong K_{2} \cong T^{m}$ for some finite simple non-abelian group $T$ and some $m \in \mathbb{Z}_{\geq 1}$.

3. $\operatorname{Soc}(G)$ is a minimal normal subgroup of $G$. Moreover, $C_{G}(\operatorname{Soc}(G))=1$ and $\operatorname{Soc}(G) \cong$ $T^{m}$ for some finite simple non-abelian group $T$ and some $m \in \mathbb{Z}_{\geq 1}$.

Theorem 5.10 (See [DM96, Theorem 1.6A]). If $G \leq \operatorname{Sym}(n)$ is a primitive permutation group and $1 \neq H \leqslant G$ is a non-trivial normal subgroup, then $H$ is transitive.

Corollary 5.11. If $p \geq 5$ is prime and $Q_{p}$ is primitive, then $\operatorname{Soc}(p) \stackrel{\text { def }}{=} \operatorname{Soc}\left(Q_{p}\right)$ acts transi- $\operatorname{Soc}(p)$ tively on $Y^{*}(p)$ and is a direct product of non-abelian simple groups.

Proof. Transitivity follows from Theorem 5.10 and the fact that the socle is a normal subgroup. Case (1) of Theorem 5.9 is ruled out because $\left|Y^{*}(p)\right|=\frac{p(p \pm 3)}{4}$ is not a prime power (or, alternatively, because $\operatorname{Aff}(p, d)$ has no non-identity elements fixing more than half of the points, such as $\left.\operatorname{rot}_{1}^{p(p+1) / 2} \in Q_{p}\right)$. So either $Q_{p}$ falls into case (2) or it falls into case (3).

We also use the following result giving strong limitations on primitive groups:

Theorem 5.12 (See [DM96, Theorems 5.3A and 5.5B]). Let $G \lessgtr \operatorname{Sym}(n), G \neq \operatorname{Alt}(n)$, be a primitive permutation group.

1. If $G$ is not 2-transitive then $|G|<\exp \left\{4 \sqrt{n}(\ln n)^{2}\right\}$.

2. If $n \geq 216$ and $G$ is 2-transitive and contains a section ${ }^{13}$ isomorphic to $\operatorname{Alt}(k)$, then $k<6 \ln n$.

Lemma 5.13. Let $p$ and $q$ be distinct primes with $Q_{p}$ and $Q_{q}$ primitive, and such that $p$ precedes $q$ in the order defined in Notation 5.2. Then $Q_{p q} \geq 1 \times \operatorname{Soc}(q)$ (sitting inside $\left.\operatorname{Sym}\left(Y^{*}(p)\right) \times \operatorname{Sym}\left(Y^{*}(q)\right)\right)$.

Proof. Recall that the primes are sorted by the order of rotation elements. So if $o_{p}\left(o_{q}\right.$, respectively) is the order of $\operatorname{rot}_{1}$ in $Q_{p}\left(Q_{q}\right.$, respectively) then $o_{p} \leq o_{q}$.

Case 1: $o_{p}<o_{q}$

If the inequality is strict, then the image of $g=\operatorname{rot}_{1}^{o_{p}} \in \Gamma$ in $Q_{p}$ is the identity whereas its image $\bar{g}$ in $Q_{q}$ is not. By Corollary 5.11, Soc $(q)$ falls under one of cases (2) or (3) from Theorem 5.9.

Assume first that $\operatorname{Soc}(q)$ falls under case (3). Since $C_{Q_{p}}(\operatorname{Soc}(q))=1$, there is some $h \in$ $\operatorname{Soc}(q)$ not commuting with $\bar{g} \in Q_{q}$, so $e \neq[\bar{g}, h]=\bar{g} h \bar{g}^{-1} h^{-1} \in \operatorname{Soc}(q) \cap \pi_{q}\left(\operatorname{ker}\left(\Gamma \rightarrow Q_{p}\right)\right)$. Since $\operatorname{Soc}(q)$ is a minimal normal subgroup of $Q_{q}$, it is generated by the conjugates of $[\bar{g}, h]$ in $Q_{q}$, all of which also belong to $\pi_{q}\left(\operatorname{ker}\left(\Gamma \rightarrow Q_{p}\right)\right)$. Thus $\operatorname{Soc}(q) \leq \pi_{q}\left(\operatorname{ker}\left(\Gamma \rightarrow Q_{p}\right)\right)$.

Now assume that $\operatorname{Soc}(q)$ falls under case (2). Since regular subgroups of $\operatorname{Sym}(n)$ are obtained as the (left or right) regular representation of a group of order $n$, every element of a regular permutation group has all its cycles with equal length. Since $\operatorname{rot}_{1} \in Q_{q}$ contains

\footnotetext{
${ }^{11}$ A permutation group $H \leq \operatorname{Sym}(n)$ is called regular if it is sharply transitive. Namely, it is transitive and free. In other words, it is transitive and of order $n$. The name originates from the observation that such subgroups are obtained as the (left or right) regular representation of order- $n$ groups.

${ }^{12}$ For $G$ a group and $K \leq G$ a subgroup, $C_{G}(K)=\{g \in G \mid g k=k g \forall k \in K\}$ is the centralizer of $K$ in $G$.

${ }^{13} \mathrm{~A}$ section of a group is some quotient of a subgroup.
} 
cycles of coprime lengths, no non-trivial power of it can belong to a regular subgroup, so $\bar{g}=\operatorname{rot}_{1}^{o_{p}} \notin K_{1} \cup K_{2}$. So there are $h_{1} \in K_{1}$ and $h_{2} \in K_{2}$ not commuting with $g$. Consider $h=h_{1} h_{2} \in K_{1} \times K_{2}=\operatorname{Soc}(q)$. Then $[\bar{g}, h]=\left(\left[\bar{g}, h_{1}\right],\left[\bar{g}, h_{1}\right]\right) \in K_{1} \times K_{2}=\operatorname{Soc}(q)$ belongs also to $\pi_{q}\left(\operatorname{ker}\left(\Gamma \rightarrow Q_{p}\right)\right)$ but not to $K_{1} \cup K_{2}$. The only normal subgroups of $Q_{p}$ which are contained in $K_{1} \times K_{2}$ are $1, K_{1}, K_{2}$ and $K_{1} \times K_{2}$. Hence $K_{1} \times K_{2}$ is generated by the conjugates in $Q_{q}$ of $[\bar{g}, h]$, all of which belong to $\pi_{q}\left(\operatorname{ker}\left(\Gamma \rightarrow Q_{p}\right)\right)$. Thus $\operatorname{Soc}(q) \leq \pi_{p}\left(\operatorname{ker}\left(\Gamma \rightarrow Q_{p}\right)\right)$.

Case 2: $o_{p}=o_{q}$

We are left with the rare case ${ }^{14}$ that $o_{p}=o_{q}$, as in $p=11$ and $q=5$. In this case $p>q$, $p \equiv 3(4), q \equiv 1(4)$ and $\left(p^{2}-1\right)=q\left(q^{2}-1\right)$. In particular, as $Q_{q}$ is primitive, it contains the full alternating group Alt $\left(Y^{*}(q)\right)$ by the CFSG-free Theorem 1.3. We claim that $Q_{p}$ has no composition factor isomorphic to $\operatorname{Alt}\left(Y^{*}(q)\right)$. Using this, we can finish as in the proof of Lemma 5.1: indeed, consider the following normal series of $Q_{p q}$

$$
1 \unlhd Q_{p q} \cap\left[1 \times \operatorname{Alt}\left(Y^{*}(q)\right)\right] \unlhd Q_{p q} \cap\left[1 \times \operatorname{Sym}\left(Y^{*}(q)\right)\right] \unlhd Q_{p q}
$$

Since $Q_{q}$ is a quotient of $Q_{p q}$, Alt (Y* (q)) is a composition factor of $Q_{p q}$, so it has to be a composition factor of one of the quotients in (24). The rightmost quotient is $Q_{p}$ which we show below has no composition factor isomorphic to $\operatorname{Alt}\left(\mathrm{Y}^{*}(\mathrm{q})\right.$ ). The second quotient is $\mathbb{Z} / 2 \mathbb{Z}$ or trivial. Thus, the leftmost quotient contains Alt ( $\mathrm{Y}^{*}(\mathrm{q})$ ) as a composition factor, namely, $Q_{p q} \geq 1 \times \operatorname{Alt}\left(\mathrm{Y}^{*}(\mathrm{q})\right)$, and we are done as $\operatorname{Soc}(q)=\operatorname{Alt}\left(\mathrm{Y}^{*}(\mathrm{q})\right)$.

So we have left to show that $Q_{p}$ has no composition factor isomorphic to $\operatorname{Alt}\left(\mathrm{Y}^{*}(\mathrm{q})\right.$ ). This is certainly the case if $Q_{p} \geq \operatorname{Alt}\left(\mathrm{Y}^{*}(\mathrm{p})\right.$ ) (as in the case $p=11, q=5$ ). So assume $Q_{p} \ngtr \operatorname{Alt}\left(Y^{*}(p)\right)$ and proceed using Theorem 5.12.

First, assume that $Q_{p}$ is not 2-transitive. Asymptotically, its order is smaller than that of Alt $\left(Y^{*}(q)\right)$ : indeed, if $n=\left|Y^{*}(p)\right|=\frac{p(p-3)}{4}$ then $n \approx q^{3}$, and so by Theorem 5.12,

$$
\begin{aligned}
\ln \left|\operatorname{Alt}\left(Y^{*}(q)\right)\right| & =\ln \left(\frac{1}{2} \cdot\left(\frac{q(q+3)}{4}\right) !\right) \approx q^{2} \ln q \\
\ln \left|Q_{p}\right| & \leq 4 \sqrt{n}(\ln n)^{2} \approx q^{1.5}(\ln q)^{2} .
\end{aligned}
$$

In fact, this asymptotic reasoning starts taking effect for $q \geq 203,897$, but for smaller values of $q$ there are no cases for which $o_{p}=o_{q}$ except for $q=5$ (this was easily verified by computer).

Finally, assume that $Q_{p}$ is 2-transitive. Then, not only does it not have a composition factor isomorphic to $\operatorname{Alt}\left(\mathrm{Y}^{*}(\mathrm{q})\right)$, it does not even have a section isomorphic to it: since $\frac{p(p-3)}{4} \geq 216$, Theorem 5.12 says that $k=\frac{q(q+3)}{4}<6 \ln \frac{p(p-3)}{4}$. This is impossible when $q \geq 13$.

We can now finish our CFSG-free proof of Lemma 5.3.

CFSG-free proof of Lemma 5.3. Assume $n=p_{1} \cdots p_{k}$ is a product of distinct primes with $Q_{p_{1}}, \ldots, Q_{p_{k}}$ primitive and $p_{1}, \ldots, p_{k}$ ordered as in Notation 5.2. We need to show that for every $j=2, \ldots, k$, the image of $\Omega_{j-1}$ in $Q_{p_{j}}, \pi_{p_{j}}\left(\Omega_{j-1}\right)$ contains a subgroup $H_{j} \leq$ $\operatorname{Sym}\left(Y^{*}\left(p_{j}\right)\right)$ which is transitive and isomorphic to a direct product of non-abelian simple groups. We show that $\pi_{p_{j}}\left(\Omega_{j-1}\right) \geq \operatorname{Soc}\left(p_{j}\right)$, which is enough by Corollary 5.11.

Without loss of generality, it is enough to prove this when $j=k$. As $\operatorname{Soc}\left(p_{k}\right) \cong \prod_{i=1}^{m} T_{i}$ with $T_{1}, \ldots, T_{m}$ non-abelian simple groups, each of them satisfies $\left[T_{i}, T_{i}\right]=T_{i}$. Hence for any $t \in \mathbb{Z}_{\geq 1}$ there is a sequence of elements $\bar{g}_{1}, \ldots, \bar{g}_{t} \in \operatorname{Soc}\left(p_{k}\right)$ so that the nested commutator

$$
\left.\left[\ldots\left[\bar{g}_{1}, \bar{g}_{2}\right], \bar{g}_{3}\right], \ldots, \bar{g}_{t}\right]
$$

\footnotetext{
${ }^{14}$ In fact, the only such case with $p<1,000,000$ is $p=5$.
} 
has non-trivial projection in each of the $T_{i}$ 's. Choose such a sequence of length $t=k-1$. By Lemma 5.13, for every $i=1, \ldots, k-1$, there is an element $g_{i} \in \Gamma$ with $\pi_{p_{i}}\left(g_{i}\right)=1$ and $\pi_{p_{k}}\left(g_{i}\right)=\bar{g}_{i}$. The element

$$
g=\left[\ldots\left[\left[g_{1}, g_{2}\right], g_{3}\right], \ldots, g_{k-1}\right] \in \Gamma
$$

satisfies then that $\pi_{p_{i}}(g)=1$ for all $i=1, \ldots, k-1$, whereas $\pi_{p_{k}}(g) \in \operatorname{Soc}\left(p_{k}\right)$ in not contained in any proper normal subgroup of $\operatorname{Soc}\left(p_{k}\right)$. Hence every element of $\operatorname{Soc}\left(p_{k}\right)$ is a product of conjugates of $\pi_{p_{k}}(g)$, and we obtain that $\pi_{p_{k}}\left(\Omega_{k-1}\right) \geq \operatorname{Soc}\left(p_{k}\right)$.

\section{$6 \quad T_{2}$-systems}

This section explains why Theorem 1.11 is equivalent to Theorems 1.3 and 1.4. Namely, if we let $\Sigma_{2,-2}(p)$ denote the set of PSL $(2, p)$-defining subgroups of $\mathrm{F}_{2}$ with associated trace -2 , our goal here is to show:

1. A one-to-one correspondence between $Y^{*}(p)$ and $\Sigma_{2,-2}(p)$, and

2. An isomorphism between $Q_{p}$, the group of permutations induced by the action of $\Gamma$ on $Y^{*}(p)$, and the group of permutations induced by the action of Aut $\left(\mathrm{F}_{2}\right)$ on $\Sigma_{2,-2}(p)$.

First, let us define $\Sigma_{2,-2}(p)$ properly. For $A, B \in \operatorname{PSL}(2, p)$, define

$$
\operatorname{Tr}(A, B) \stackrel{\text { def }}{=}(\operatorname{tr} A, \operatorname{tr} B, \operatorname{tr} A B) \in \mathbb{F}_{p}^{3} / \sim,
$$

where $\sim$ is the equivalence of changing the sign of two of the coordinates (each of $A$ and $B$ is a well-defined matrix in SL $(2, p)$ up to a sign). Assume $\langle A, B\rangle=\operatorname{PSL}(2, p)$, and let $\varphi: \mathrm{F}_{2} \rightarrow \mathrm{PSL}(2, p)$ be the epimorphism mapping the generators $a$ and $b$ of $\mathrm{F}_{2}$ to $A$ and $B$, respectively. The kernel $N=\operatorname{ker} \varphi$ is a PSL $(2, p)$-defining subgroup of $\mathrm{F}_{2}$, and define

$$
\operatorname{Tr}(N) \stackrel{\text { def }}{=} \operatorname{Tr}(A, B) .
$$

Recall that $\Sigma_{2}(G)$ denotes the set of $G$-defining subgroups of $\mathrm{F}_{2}$.

Claim 6.1. The map Tr: $\Sigma_{2}(\operatorname{PSL}(2, p)) \rightarrow \mathbb{F}_{p}^{3} / \sim$ is well-defined.

Proof. Let $G=\operatorname{PSL}(2, p)$. Given $N \in \Sigma_{2}(G)$, all epimorphisms $\mathrm{F}_{2} \rightarrow G$ with kernel $N$ are obtained one from the other by post-composition with some automorphism from Aut $(G)$. But every automorphism of $G$ is obtained by a conjugation by some element from PGL $(2, p)$. Evidently, such conjugation does not effect the image of $\operatorname{Tr}$ on the images of the generators $a$ and $b$ of $\mathrm{F}_{2}$.

Recall that $\operatorname{tr}([A, B])=Q(\operatorname{tr} A, \operatorname{tr} B, \operatorname{tr} A B)$ where $Q(x, y, z)=x^{2}+y^{2}+z^{2}-x y z-2$. Thus, for $N \in \Sigma_{2}(\operatorname{PSL}(2, p))$, the element

$$
Q(N) \stackrel{\text { def }}{=} Q(\operatorname{Tr}(N)) \in \mathbb{F}_{p}
$$

is well-defined, and we denote

$$
\Sigma_{2,-2}(p) \stackrel{\text { def }}{=} Q^{-1}(-2) \subseteq \Sigma_{2}(\operatorname{PSL}(2, p))
$$

Note that, by definition, for every $N \in \Sigma_{2,-2}(p)$ the triple $\operatorname{Tr}(N)$ is (an equivalence class up to sign changes of) a solution to the Markoff equation (1) over $\mathbb{Z} / p \mathbb{Z}$. 
Claim 6.2. The map $\left.\operatorname{Tr}\right|_{\Sigma_{2,-2}(p)}$ is a bijection from $\Sigma_{2,-2}(p)$ to $Y^{*}(p)$.

Proof. Consider the map $\widetilde{\operatorname{Tr}}: \mathrm{SL}(2, p) \times \mathrm{SL}(2, p) \rightarrow \mathbb{F}_{p}^{3}$ defined as in (25). By [Mac69, Theorems 2 and 3], if $(x, y, z) \in \mathbb{F}_{p}^{3}$ is the image of some generating pair in $\operatorname{SL}(2, p)$, then every two pairs in $\widetilde{\operatorname{Tr}}^{-1}((x, y, z))$ are conjugated one to the other by an element $g \in \mathrm{SL}\left(2, \overline{\mathbb{F}_{p}}\right)$. Since these pairs are generating, this conjugation by $g$ is an automorphism of SL $(2, p)$. As every automorphism of $\mathrm{SL}(2, p)$ is also an automorphism of PSL $(2, p)$, we obtain that

$$
\left.\operatorname{Tr}\right|_{\Sigma_{2,-2}(p)}: \Sigma_{2,-2}(p) \rightarrow \mathbb{F}_{p}^{3} / \sim
$$

is injective.

By [Mac69, Thm 1], the map $\widetilde{T r}$ is surjective. The analysis in [MW13, Section 11] shows that the only triple $(x, y, z) \in \mathbb{F}_{p}^{3}$ with $Q(x, y, z)=-2$ which does not correspond to generating pairs is ${ }^{15}(0,0,0)$. This completes the proof of the claim.

We have left to show the isomorphism of $Q_{p}$ and the permutation group induced by Aut $\left(\mathrm{F}_{2}\right)$ on $Y^{*}(p) \cong \Sigma_{2,-2}(p)$. Recall that $Q_{p}=\left\langle\tau_{(12)}, \tau_{(23)}, R_{3}\right\rangle$. For $\mathrm{F}_{2}=\mathrm{F}(a, b)$, Aut $\left(\mathrm{F}_{2}\right)$ is generated by the following Nielsen moves ${ }^{16}: r:(a, b) \mapsto\left(a^{-1}, b\right), s:(a, b) \mapsto(b, a)$ and $t:(a, b) \mapsto\left(a^{-1}, a b\right)$. The induced action of these three automorphisms on $Y^{*}(p)$ is easily seen to be the same action given by $R_{3}, \tau_{(12)}$ and $\tau_{(23)}$, respectively.

\section{Appendix}

\section{A On the order of a quadratic integer modulo most primes By Dan Carmon}

Throughout this appendix, we use the notation $f \ll g$ to mean that there exists an absolute constant $C>0$ for which $f \leq C g$ for all valid values of the implicit variables. The similar notation $f \ll_{a} g$ means there exists a function $C=C(a)>0$ for which $f \leq C g$. The notation $f \asymp g$ is shorthand for " $f \ll g$ and $g \ll f$ ".

\section{The main claim}

Let $a \in \mathbb{Q}(\sqrt{D})$ be a fixed quadratic integer with norm 1 and absolute value $|a|>1$ (e.g. $\left.a=\frac{3+\sqrt{5}}{2}\right)$. For primes $p \nmid D$, consider the residue $\bar{a}=(a \bmod p)$, as an element of either $\mathbb{F}_{p}$ or $\mathbb{F}_{p^{2}}$, depending on whether $D$ is a quadratic residue modulo $p$. In both cases there are two possible choices for $\bar{a}$, but its order $o_{p}(a)$, which is the smallest positive integer satisfying $\bar{a}^{o_{p}(a)}=1 \in \mathbb{F}_{p^{2}}$ is well-defined. Let $\pi(x)=\#\{p \leq x: p$-prime $\}$ be the prime counting $\pi(x)$ function. We prove the following claim:

Proposition A.1. For any constant $C \geq 1$,

$$
\#\left\{p \leq x: o_{p}(a) \leq C \sqrt{x}\right\} \ll_{a} \frac{\pi(x)}{(\log x)^{\delta}(\log \log x)^{3 / 2-\delta}},
$$

\footnotetext{
${ }^{15}$ To see that $(0,0,0)$ is not associated with a generating pair, note that if $A \in \mathrm{PSL}(2, p)$ has trace 0 , then $A$ is an involution. If both $A$ and $B$ are involutions, then $\langle A, B\rangle$ is a dihedral group, which is a proper subgroup of $\operatorname{PSL}(2, p)$.

${ }^{16}$ We deliberately copy the notation for these Nielsen moves from [MW13].
} 
where $\delta$ is the Erdôs-Tenenbaum-Ford constant,

$$
\delta=1-\frac{1+\log \log 2}{\log 2}=0.086071 \ldots
$$

In particular, the set of primes with $o_{p}(a)>C \sqrt{p}$ has relative density 1.

\section{Proof outline}

Proposition A.1 follows from the combination of two sub-lemmas:

Lemma A.2. Let $\alpha=\alpha(x)$ tend to infinity arbitrarily slowly with $x$, and let $y=\sqrt{\frac{x}{\alpha}}$. Then

$$
\#\left\{p \leq x: o_{p}(a) \leq y\right\} \ll_{a} \frac{\pi(x)}{\alpha} .
$$

Lemma A.3. Let $\alpha, y$ be as in the previous Lemma. Define $z=C \sqrt{x}$, and $u_{0}=\frac{\log \alpha}{\log x}$. Suppose further that $\alpha \in\left(\frac{4}{C^{2}}, \frac{\sqrt{x}}{C}\right)$. Then

$$
\#\{p \leq x: \exists d \in(y, z], p \equiv \pm 1(\bmod d)\} \ll u_{0}^{\delta}\left(\log \frac{2}{u_{0}}\right)^{-3 / 2} \pi(x) .
$$

Indeed, since $a$ has norm $1, o_{p}(a)$ is always a factor of either $p-1$ when $D$ is a quadratic residue modulo $p$, or of $p+1$ when $D$ is a non-quadratic residue, i.e. $p \equiv \pm 1\left(\bmod o_{p}(a)\right)$ in either case. Thus $o_{p}(a) \leq C \sqrt{x}$ implies that $p$ is either included in the set of the first lemma if $o_{p}(a) \leq y$, or in the set of the second lemma if $o_{p}(a) \in(y, z]$. Choosing the optimal value

$$
\alpha=(\log x)^{\delta}(\log \log x)^{3 / 2-\delta}
$$

yields the claimed value in the right hand side of both lemmas.

\section{Proofs of the lemmas}

Proof of Lemma A.2. The following proof is an adaptation of an argument from Erdôs and Murty [EM99, Introduction], in which only integral values $a$ and a specific choice of $\alpha$ were considered.

For every $k \geq 1$ define $A_{k}=\frac{a^{k}-a^{-k}}{\sqrt{D}}$. Note that $A_{k}$ is always an integer, with $\left|A_{k}\right|<|a|^{k}$, and that $o_{p}(a)=k$ implies $p \mid A_{k}$. Define

$$
B_{y}=\prod_{k=1}^{\lfloor y\rfloor} A_{k}
$$

so that $o_{p}(a) \leq y$ implies $p \mid B_{y}$. We now observe that

$$
\log B_{y}=\sum_{k=1}^{\lfloor y\rfloor} \log A_{k} \leq \sum_{k=1}^{\lfloor y\rfloor} k \log |a| \ll_{a} y^{2}=\frac{x}{\alpha},
$$

and on the other hand

$$
\begin{aligned}
\log B_{y} & \geq \sum_{p \mid B_{y}} \log p \geq \sum_{p: o_{p}(a) \leq y} \log p \geq \sum_{\substack{\sqrt{x}<p \leq x \\
o_{p}(a) \leq y}} \log \sqrt{x} \\
& =\frac{1}{2} \log x \cdot \#\left\{\sqrt{x}<p \leq x: o_{p}(a) \leq y\right\},
\end{aligned}
$$


whence

$$
\#\left\{p \leq x: o_{p}(a) \leq y\right\} \leq \pi(\sqrt{x})+\frac{2 \log B_{y}}{\log x} \ll_{a} \frac{2}{\alpha} \frac{x}{\log x} \ll \frac{\pi(x)}{\alpha}
$$

Proof of Lemma A.3. This lemma is a direct application of results due to Ford [For08]. We cite the relevant definitions and theorems. Ford's main object of study is the function

$$
H(x, y, z)=\#\{n \leq x: \exists d \in(y, z], d \mid n\} .
$$

We are particularly interested in the specialized function

$$
H\left(x, y, z ; P_{\lambda}\right)=\#\left\{n \leq x: n \in P_{\lambda}, \exists d \in(y, z], d \mid n\right\},
$$

where $P_{\lambda}=\{p+\lambda: p$ - prime $\}$ is a set of shifted primes, and more specifically only for $\lambda= \pm 1$.

In [For08, Theorem 1], Ford estimates $H(x, y, z)$ for all possible choices of $y \leq z \leq x$. The relevant case for our choice of $y, z$ is the third subcase of case (v), wherein $x, y, z$ are all large, $y \leq \sqrt{x}$, and $z \in\left[2 y, y^{2}\right]$, all of which are immediately validated for our values, due to the constraint on $\alpha$. For this case, the theorem states

$$
\frac{H(x, y, z)}{x} \asymp u^{\delta}\left(\log \frac{2}{u}\right)^{-3 / 2}
$$

where $u$ is the number satisfying $z=y^{1+u}$, or equivalently

$$
u=\frac{\log (z / y)}{\log y}=\frac{\log (C \sqrt{\alpha})}{\log (\sqrt{x / \alpha})}=\frac{\log \alpha+2 \log C}{\log x-\log \alpha} \asymp \frac{\log \alpha}{\log x}=u_{0} .
$$

In [For08, Theorem 6], Ford estimates $H\left(x, y, z ; P_{\lambda}\right)$, for any fixed non-zero $\lambda$. The behaviour of the function is determined by whether $z$ is greater or lesser than $y+(\log y)^{2 / 3}$. The constraint on $\alpha$ implies $z \geq 2 y$, so we are certainly in the regime of $z \geq y+(\log y)^{2 / 3}$, in which the theorem yields

$$
H\left(x, y, z ; P_{\lambda}\right) \ll_{\lambda} \frac{H(x, y, z)}{\log x} .
$$

Combining the estimates (33),(34),(35) yields (28), proving the lemma.

\section{References}

[BGS16] Jean Bourgain, Alexander Gamburd, and Peter Sarnak. Markoff triples and strong approximation. Comptes Rendus Mathematique, 354(2):131-135, 2016.

[BGS17] Jean Bourgain, Alexander Gamburd, and Peter Sarnak. Markoff surfaces and strong approximation: 1. arXiv preprint arXiv:1607.01530, 2017+.

[CGMP16] Alois Cerbu, Elijah Gunther, Michael Magee, and Luke Peilen. The cycle structure of a Markoff automorphism over finite fields. preprint arXiv:1610.07077, 2016.

[DM96] John D. Dixon and Brian Mortimer. Permutation groups. Springer Science \& Business Media, 1996.

[EM99] Pál Erdős and M. Ram Murty. On the order of a $(\bmod \mathrm{p})$. CRM Proceedings and Lecture Notes, 19:87-97, 1999. 
[Eva93] Martin J Evans. T-systems of certain finite simple groups. Mathematical Proceedings of the Cambridge Philosophical Society, 113(1):9-22, 1993.

[For08] Kevin Ford. The distribution of integers with a divisor in a given interval. Annals of mathematics, 168(2):367-433, 2008.

[Gil77] Robert Gilman. Finite quotients of the automorphism group of a free group. Canad. J. Math, 29(3):541-551, 1977.

[GM98] Robert Guralnick and Kay Magaard. On the minimal degree of a primitive permutation group. Journal of Algebra, 207(1):127-145, 1998.

[GS09] Shelly Garion and Aner Shalev. Commutator maps, measure preservation, and Tsystems. Transactions of the American Mathematical Society, 361(9):4631-4651, 2009.

[Lub11] Alexander Lubotzky. Dynamics of Aut(Fn) actions on group presentations and representations. In B. Farb and D. Fisher, editors, Geometry, Rigidity, and Group Actions, pages 609-643. Chicago University press, 2011.

[Mac69] Alexander M. Macbeath. Generators of the linear fractional groups. In Proc. Symp. Pure Math, volume 12, pages 14-32, 1969.

[Mar79] Andrey Markoff. Sur les formes quadratiques binaires indéfinies. Mathematische Annalen, 15(3):381-406, 1879.

[Mar80] Andrey Markoff. Sur les formes quadratiques binaires indéfinies. Mathematische Annalen, 17(3):379-399, 1880.

[MW13] Darryl McCullough and Marcus Wanderley. Nielsen equivalence of generating pairs of SL(2, q). Glasgow Mathematical Journal, 55(03):481-509, 2013.

[Pak01] Igor Pak. What do we know about the product replacement algorithm? In W. Kantor and A. Seress, editors, Groups and Computation III, pages 301-347. de Gruyter, 2001.

[Sch76] Wolfgang M. Schmidt. Equations over finite fields: An elementary approach. Springer-Verlag, Halsted Press, 1976.

Chen Meiri,

Department of Mathematics,

Technion - Israel Institute of Technology

Haifa 32000 Israel

chenm@tx.technion.ac.il

Doron Puder,

School of Mathematical Sciences,

Tel-Aviv University,

Tel-Aviv 69978 Israel

doronpuder@gmail.com

Dan Carmon,

School of Mathematical Sciences,

Tel-Aviv University,

Tel-Aviv 69978 Israel

dancarmo@post.tau.ac.il 